\title{
LIM kinase/cofilin dysregulation promotes macrothrombocytopenia in severe von Willebrand disease-type 2B
}

\author{
Alexandre Kauskot, ${ }^{1,2}$ Sonia Poirault-Chassac, ${ }^{2}$ Frédéric Adam, ${ }^{1}$ Vincent Muczynski, ${ }^{1}$ \\ Gabriel Aymé, ${ }^{1}$ Caterina Casari, ${ }^{1}$ Jean-Claude Bordet, ${ }^{3,4}$ Christelle Soukaseum, ${ }^{1}$ Chantal Rothschild, ${ }^{5}$ \\ Valérie Proulle, ${ }^{1,6}$ Audrey Pietrzyk-Nivau, ${ }^{2}$ Eliane Berrou, ${ }^{1}$ Olivier D. Christophe, ${ }^{1}$ \\ Jean-Philippe Rosa, ${ }^{1}$ Peter J. Lenting, ${ }^{1}$ Marijke Bryckaert, ${ }^{1}$ Cécile V. Denis, ${ }^{1}$ and Dominique Baruch ${ }^{2}$ \\ 1INSERM UMR-S 1176, Univ Paris-Sud, Université Paris-Saclay, 94276 Le Kremlin-Bicêtre, France. INSERM UMR-S 1140, \\ Univ Paris Descartes, Sorbonne Paris Cité, Paris, France. ${ }^{3}$ Laboratoire d'Hémostase, Hôpital Edouard Herriot, Lyon, France. \\ ${ }^{4}$ Laboratoire de Recherche sur l'Hémophilie, UCBL1, Faculté de Médecine Lyon-Est, Lyon, France. ${ }^{5}$ Centre Hémophilie, \\ Hôpital Necker, Paris, France. ㅁepartment of Biological Hematology, CHU Bicêtre, Hôpitaux Universitaires Paris Sud, \\ AP-HP, Paris, France.
}

von Willebrand disease type 2B (VWD-type 2B) is characterized by gain-of-function mutations of von Willebrand factor (VWF) that enhance its binding to platelet glycoprotein lba and alter the protein's multimeric structure. Patients with VWD-type 2B display variable extents of bleeding associated with macrothrombocytopenia and sometimes with thrombopathy. Here, we addressed the molecular mechanism underlying the severe macrothrombocytopenia both in a knockin murine model for VWD-type 2B by introducing the p.V1316M mutation in the murine Vwf gene and in a patient bearing this mutation. We provide evidence of a profound defect in megakaryocyte (MK) function since: (a) the extent of proplatelet formation was drastically decreased in 2B MKs, with thick proplatelet extensions and large swellings; and (b) 2B MKs presented actin disorganization that was controlled by upregulation of the RhoA/LIM kinase (LIMK)/cofilin pathway. In vitro and in vivo inhibition of the LIMK/cofilin signaling pathway rescued actin turnover and restored normal proplatelet formation, platelet count, and platelet size. These data indicate, to our knowledge for the first time, that the severe macrothrombocytopenia in VWD-type 2B p.V1316M is due to an MK dysfunction that originates from a constitutive activation of the RhoA/LIMK/cofilin pathway and actin disorganization. This suggests a potentially new function of vWF during platelet formation that involves regulation of actin dynamics.

Authorship note: S. Poirault-Chassac and F. Adam contributed equally to this work. C.V. Denis and D. Baruch are co-senior authors on this work.

Conflict of interest: The authors have declared that no conflict of interest exists.

Submitted: May 19, 2016 Accepted: September 1, 2016 Published: October 6, 2016

Reference information: JCI Insight. 2016;1(16):e88643. doi:10.1172/jci.insight.88643.

\section{Introduction}

Remodeling of the cytoskeleton is pertinent to the morphological changes that cells have to endure during processes such as cytokinesis, cell polarization, and migration. Actin filament disassembly is a dynamic event involving many cellular components, and among the known filamentous actin (F-actin) severing proteins, the members of the actin-depolymerizing factor (ADF)/cofilin family play a pivotal role by spatially and temporally regulating actin turnover. The activity of ADF and cofilin is tightly regulated via (de)phosphorylation of a regulatory serine residue. Upon dephosphorylation, ADF/cofilin associates preferentially in a cooperative manner with ADP-bound F-actin (1). This binding induces a twist in the actin filament and enhances actin disassembly by filament severing and by dissociation of actin monomers (reviewed in ref. 2). Major reorganizations of the cytoskeleton are also required when mature megakaryocytes (MKs) proceed to generate proplatelets, an event that precedes liberation of platelets in the blood stream (3). In the initial phase of this process, MKs extend their demarcation membranes to form proplatelets. Subsequently, additional changes in cytoskeletal structures cause proplatelets to extend further and allow the relocalization of organelles $(4,5)$. Actin cytoskeleton and microtubules are involved in proplatelet extension and in the final abscission and cleavage leading to platelet formation in the blood stream of marrow sinusoids (5-7). In vivo, actin filament turnover is a critical step in the terminal phase of platelet formation, as well as in the maturation and sizing of platelets to produce the homogeneous mature platelet population $(6,8)$. 
Table 1. Hematologic parameters ${ }^{A}$

\begin{tabular}{|c|c|c|}
\hline & WT & 2B \\
\hline Platelets $\left(\times 10^{3} / \mu \mathrm{l}\right)$ & $930 \pm 25$ & $466 \pm 27^{B}$ \\
\hline MPV (fl) & $5.43 \pm 0.05$ & $8.53 \pm 0.11^{\mathrm{B}}$ \\
\hline $\mathrm{RBC}\left(\times 10^{6} / \mu \mathrm{l}\right)$ & $9.75 \pm 0.18$ & $9.58 \pm 0.20$ \\
\hline MCV (fI) & $50.5 \pm 0.3$ & $49.8 \pm 0.3$ \\
\hline $\mathrm{Hgb}(\mathrm{g} / \mathrm{dl})$ & $15.4 \pm 0.3$ & $15.1 \pm 0.3$ \\
\hline Hct (\%) & $49 \pm 1$ & $47 \pm 1$ \\
\hline WBC $\left(\times 10^{3} / \mu \mathrm{l}\right)$ & $5.7 \pm 0.5$ & $6.8 \pm 0.5$ \\
\hline Lymphocytes $\left(\times 10^{3} / \mu \mathrm{l}\right)$ & $5.1 \pm 0.5$ & $6.3 \pm 0.4$ \\
\hline TPO $(\mathrm{pg} / \mathrm{ml})^{\mathrm{C}}$ & $303 \pm 19$ & $488 \pm 24^{B}$ \\
\hline \multicolumn{3}{|c|}{$\begin{array}{l}{ }^{A} \text { For blood cell parameters, WT } n=30 \text { and } 2 B n=29 .{ }^{B} P<0.001 \\
\text { as determined by Student's } t \text { test. }{ }^{C} \text { For TPO measurement, WT } \\
n=8 \text { and } 2 B n=6 \text {. Hct, hematocrit; Hgb, hemoglobin; MCV, } \\
\text { mean corpuscular volume; MPV, mean platelet volume; RBC, } \\
\text { red blood cells; TPO, thrombopoietin; WBC, white blood cells. }\end{array}$} \\
\hline
\end{tabular}

Interestingly, in many disorders characterized by macrothrombocytopenia the underlying mechanism remains unclear. A noticeable example thereof is von Willebrand disease (VWD)-type 2B, a bleeding disorder caused by mutations in the gene encoding von Willebrand factor (vWF). vWF is a circulating multimeric protein expressed in endothelial cells and MKs that plays a crucial role in the recruitment of platelets to sites of vascular injury via interactions with the platelet receptor glycoprotein Ib $\alpha$ (GPIb $\alpha$ ). In VWD-type 2B, gainof-function mutations in the GPIb $\alpha$-binding A1 domain of vWF increase the affinity for this platelet receptor, provoking spontaneous interactions between vWF and platelets and loss of high-molecular-weight vWF multimers (9). The underlying causes of thrombocytopenia in VWD-type $2 \mathrm{~B}$ are multiple, such as the incorporation of platelets bound to plasma $\mathrm{vWF}$ into circulating aggregates that display an increased platelet clearance $(10,11)$. While platelet apoptosis does not seem to be involved (12), defective platelet production appears central to the mechanism of thrombocytopenia in VWD-type 2B. Recently, it became clear that the vWF-GPIb $\alpha$ interaction might play a role in megakaryocytopoiesis with the inventory of macrothrombocytopenic VWD-type 2B patients (13). Indeed, the presence of giant platelets in VWD-type 2B was suggested to result from enhanced binding of endogenous vWF to GPIb $\alpha$ of MKs (14). However, no insight into the molecular mechanisms that could lead to such a dramatic effect on platelet production was provided. The severity of VWD-type $2 \mathrm{~B}$ is

strikingly mutation dependent and one of the most severe mutations is the p.V1316M replacement, which is associated with a constant thrombocytopenia and the presence of giant platelets (15). Interestingly, in a model of platelet formation upregulated by exposure of cultured MKs on a vWF surface to high shear forces, we have previously established a defect in platelet release from normal MKs exposed to immobilized p.V1316M vWF (16). We have recently engineered a knockin murine model for severe VWD-type 2B by introducing the p.V1316M mutation in the A1 domain of the $V w f$ gene, and we have demonstrated that these mice display a bleeding phenotype that we attributed to both macrothrombocytopenia and thrombopathy (17). Given the crucial role of the cytoskeletal changes to allow megakaryocytopoiesis, we used this mouse model of VWD-type $2 \mathrm{~B}$ to investigate the hypothesis that a disturbed regulation of actin remodeling contributes to impaired proplatelet formation in VWD-type 2B. Our studies reveal a dysregulation of LIM kinase (LIMK)/cofilin-dependent disassembly of actin filaments in VWD-type 2B. Inhibition of LIMK rescues proplatelet formation in vitro and increases platelet counts to near-normal levels in vivo. Altogether, our results indicate that expression of the type 2B mutant vWF/p.V1316M severely disturbs the signaling pathways in MKs required for platelet formation, thereby contributing to the macrothrombocytopenia associated with this disorder.

\section{Results}

The type $2 B$ mutant $v W F / p$.V1316M induces macrothrombocytopenia in mice. The presence of giant platelets in type 2B mutant vWF/p.V1316M (hereafter referred to simply as 2B) mice has been previously associated with reduced platelet counts (17). Platelet parameters of 2B mice are depicted in Table 1: the mean platelet volume (MPV) was increased by $44 \%$ compared with WT. Blood smear staining (Figure 1A), and transmission electron microscopy (TEM) (Figure 1B and Supplemental Figure 1; supplemental material available online with this article; doi:10.1172/jci.insight.88643DS1) confirmed the presence of giant platelets. The number of $\alpha$-granules in platelets was similar in both groups (Supplemental Figure 1). In $2 \mathrm{~B}$ mice, the average platelet count was decreased by $50 \%$ compared with WT mice $\left(466 \pm 27 \times 10^{3}\right.$ platelets $/ \mu 1$ in $2 \mathrm{~B}$ vs. $930 \pm 25 \times 10^{3}$ platelets $/ \mu 1$ in WT, $P<0.001$; Table 1 ), but the number of MKs was not different in the bone marrow (BM) of $2 \mathrm{~B}$ mice compared with WT mice, suggesting that the thrombocytopenia did not result from a reduced number of $\mathrm{MKs}$ (Figure 1C). Other blood cell counts were similar between 2B and WT mice (Table 1). We also investigated the expression of vWF in MKs. vWF expression was restricted to $\alpha$-granules in WT and $2 \mathrm{~B}$ MKs from BM or those obtained after cell culture in permeabilized conditions (Figure 1D), and was not observed at the surface of nonpermeabilized mature MKs (Supplemental Figure 2). Altogether, these data suggest that the features of the macrothrombocytopenia observed in human VWD-type 2B are reproduced in mice expressing mutant vWF/p.V1316M. 
A

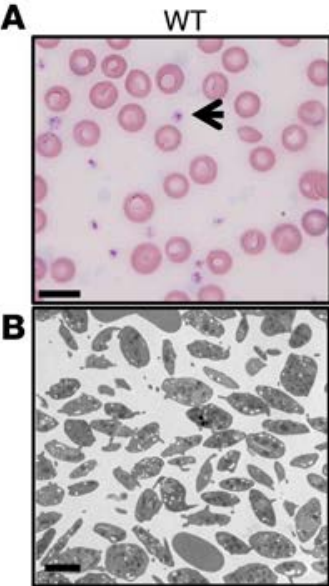

D

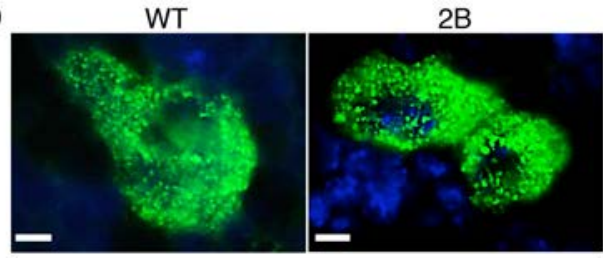

Bone marrow
C

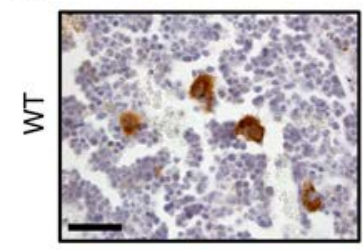

$\stackrel{\mathscr{N}}{2}$
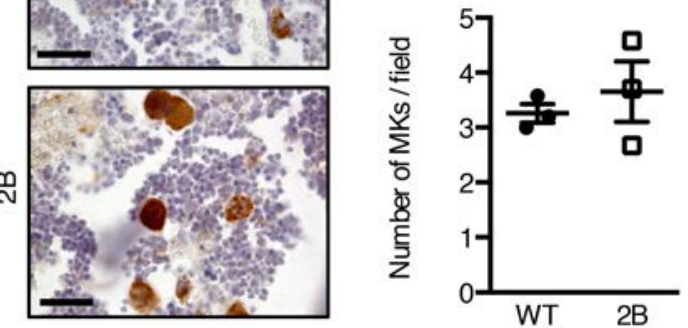

Figure 1. Platelet volume and vWF expression in megakaryocytes (MKs) of the murine model type 2B mutant vWF/p.V1316M (2B). (A) Representative blood smears of WT and 2B mice. Platelets are shown with arrows. Scale bars: $10 \mu \mathrm{m}$. (B) Transmission electron microscopy of WT and 2B platelets. Scale bars: $2 \mu \mathrm{m}$. (C) Representative images of MKs (VWF staining [brown] plus H\&E) within the bone marrow and quantification of the number of MKs/field in 3 different mice in each genotype. Scale bars: $30 \mu \mathrm{m}$. Statistical significance was determined by Student's $t$ test $(P<0.05)$. (D) Representative images of vWF expression in MKs within the bone marrow or from cell culture. Scale bars: $10 \mu \mathrm{m}$.

$2 B$ mice exhibit abnormal $M K$ proplatelet extensions and platelet production. The observation that platelet count is reduced in $2 \mathrm{~B}$ mice suggests that the presence of mutant $\mathrm{vWF}$ inside MKs might reduce their platelet production. An indicator of platelet production in vivo is platelet recovery following antibody-mediated depletion (18). Depletion of circulating platelets by administration of anti-mouse GPIb $\alpha$ antibodies resulted in a similar thrombocytopenia for both WT and $2 \mathrm{~B}$ mice 48 hours after injection $\left(33 \pm 4 \times 10^{3}\right.$ platelets $/ \mu 1$ vs. 50 $\pm 12 \times 10^{3}$ platelets $/ \mu 1$, respectively). A significant delay in platelet count recovery was observed in $2 \mathrm{~B}$ mice (Figure 2A). For instance, 72 hours after depletion, platelet count was $97 \pm 24 \times 10^{3}$ platelets $/ \mu 1$ in $2 \mathrm{~B}$ mice vs. $288 \pm 69 \times 10^{3}$ platelets $/ \mu 1$ in WT mice $(P<0.01)$. In WT mice, a transient increase in mean platelet volume (MPV) was measured during the recovery, followed by a return to normal, suggesting the production of newly formed platelets. In contrast, MPV remained unchanged during the recovery in 2B mice, suggesting an intrinsic inability of these mice to produce platelets with normal MPV (Figure 2A). We then evaluated the proplatelet formation in a native microenvironment as previously described (19). In WT BM, distinct morphological features of MKs included those with a spherical shape, others that were deformed, or developed thick extensions (proplatelets) and a decreased cell body size (Supplemental Figure 3). In contrast, in 2B BM, major alterations were observed, since the number of $2 \mathrm{~B} \mathrm{MKs}$ forming proplatelets was drastically decreased compared with WT $(1.7 \% \pm 1.1 \%$ vs. $28 \% \pm 11 \%$ in $2 \mathrm{~B}$ and WT mice, respectively), while $62 \% \pm 7 \%$ of $2 \mathrm{~B}$ MKs retained a round shape compared with $32 \% \pm 4 \%$ in WT mice (Figure $2 \mathrm{~B}$ ). Functional responses of $2 \mathrm{~B}$ MKs were then analyzed in vitro with cultured BM-derived MKs on fibrinogen-coated surfaces, as previously described $(7,20)$. The number of $2 \mathrm{~B} \mathrm{MKs}$ undergoing proplatelet formation was significantly reduced compared with WT MKs $(17 \% \pm 5 \%$ vs. $45 \% \pm 6 \%, P<0.05)$ (Figure $2, \mathrm{C}$ and D). Furthermore, the number of proplatelets per MK was also decreased for $2 \mathrm{~B}$ MKs $(3.6 \pm 1.1$ vs. $8.8 \pm 1.6$ for WT MKs, $P<0.05)$ (Figure $2, \mathrm{C}$ and D). Interestingly, the average diameter of platelet-like structures occurring along the proplatelets was increased in 2B MKs $(17 \pm 3 \mu \mathrm{m})$ versus WT MKs $(3 \pm 1 \mu \mathrm{m}, P<0.01)$ (Figure 2D). Because platelets are released in the circulation following rupture of proplatelets, we analyzed the capacity of MKs to release platelets in a flow chamber model over a vWF matrix as previously described $(16,21)$. WT MKs were able to form proplatelets and release platelets in the flow (Figure 2E and Supplemental Movie 1). By contrast, 2B MKs 
A

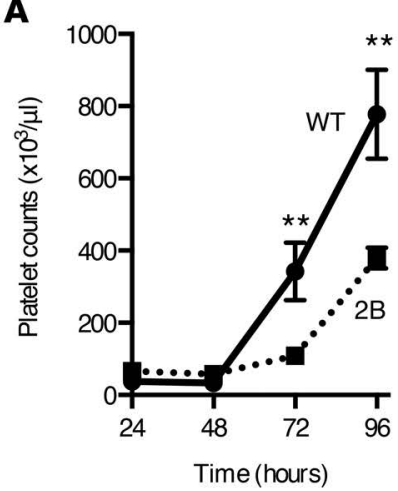

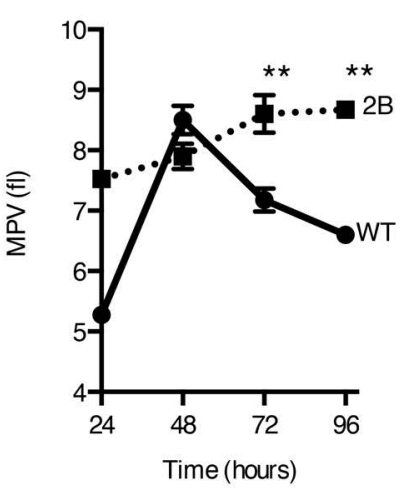

B

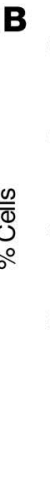

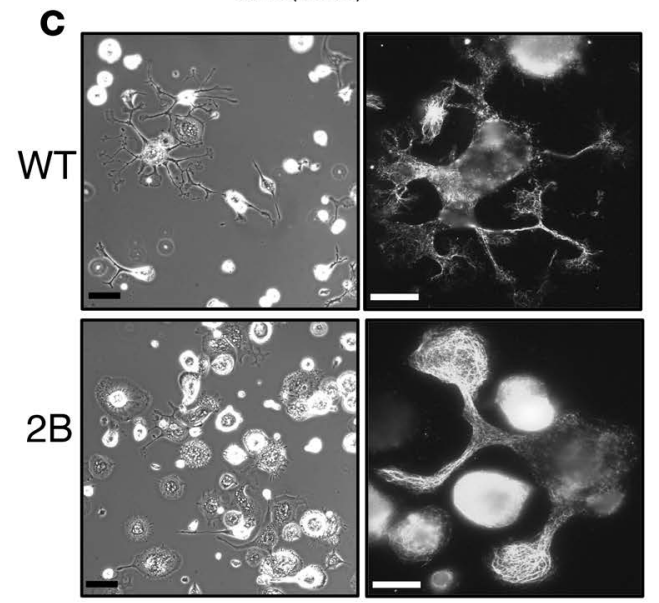

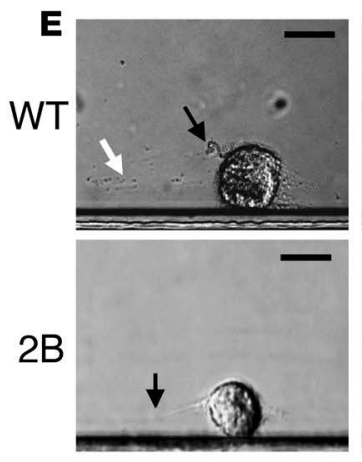

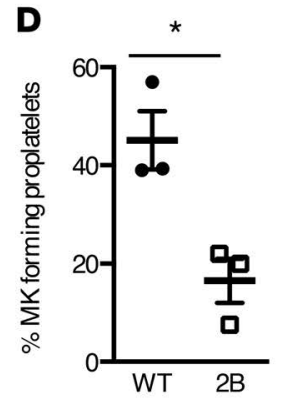
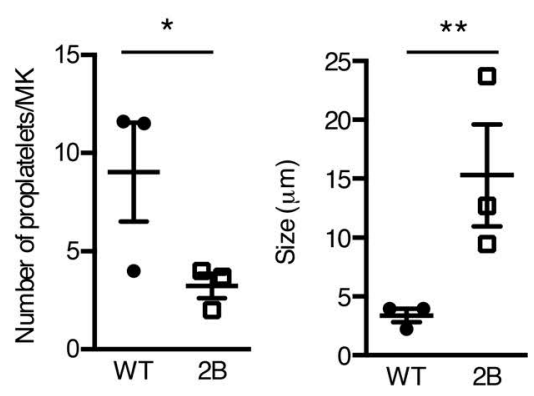

Figure 2. Platelet/proplatelet formation in vivo and in vitro is affected by the p.V1316M mutation. (A) Whole-blood platelet counts of WT (solid line) or type 2B mutant vWF/p.V1316M (2B, dashed line) mice were obtained after immune-induced thrombocytopenia following intravenous injection of antimouse GPIb $\alpha$ antibodies. Platelet counts (left) and mean platelet volume (MPV) (right) were measured at indicated times ( $n=9$ for WT and 6 for 2B). Statistical significance was determined by 1-way ANOVA followed by Dunnett's test. ${ }^{* *} P<0.01$. (B) Quantification of shape change of megakaryocytes (MKs) in bone marrow explant experiments measured in 3 separate experiments (75-125 MKs were analyzed/experiment). (C) Mature MKs were incubated over a fibrinogen matrix for 5 hours. Representative images of MKs (left, scale bars: $50 \mu \mathrm{m}$ ) and representative images of $\alpha$-tubulin staining in MKs (right, scale bars: $25 \mu \mathrm{m}$ ). (D) Quantification of the percentage of MKs forming proplatelets (left), the number of proplatelets/MK (middle), and the size of platelet-like structures (right) measured in 3 separate experiments. Statistical significance was determined by Student's $t$ test. ${ }^{*} P<0.05$, ${ }^{* *} P<0.01(20-45$ MKs were analyzed/experiment). (E) Representative images of WT and 2B MKs forming proplatelets during perfusion over a vWF matrix at a shear stress of 18 dyn/ $\mathrm{cm}^{2}$. Black arrows indicate proplatelets attached to the cell, and white arrows indicate platelets and proplatelets released from the MKs. Scale bars: $20 \mu \mathrm{m}$. Graph of the quantification of the number of platelets and proplatelets released in the flow chamber during perfusion of mature MKs at 10, 20, 30 , and 40 minutes over a VWF matrix. The number of platelets and proplatelets released from MKs and attached in the flow chamber was measured in 3 separate experiments (right). Statistical significance was determined by 1-way ANOVA followed by Dunnett's test. ${ }^{* *} P<0.01$.

formed few proplatelets and the rupture process was impaired, with the proplatelet extension failing to break and remaining attached to the cell body (Figure 2E and Supplemental Movie 2). Our data thus demonstrate that the last steps of platelet production are impaired in $2 \mathrm{~B} \mathrm{MKs}$, consistent with a reduced rate of platelet production and the presence of giant platelets in vivo. 


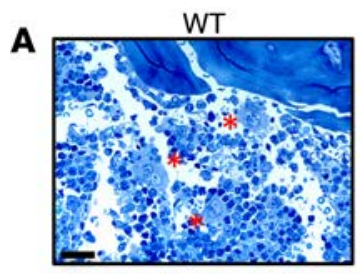

$\mathbf{B}$
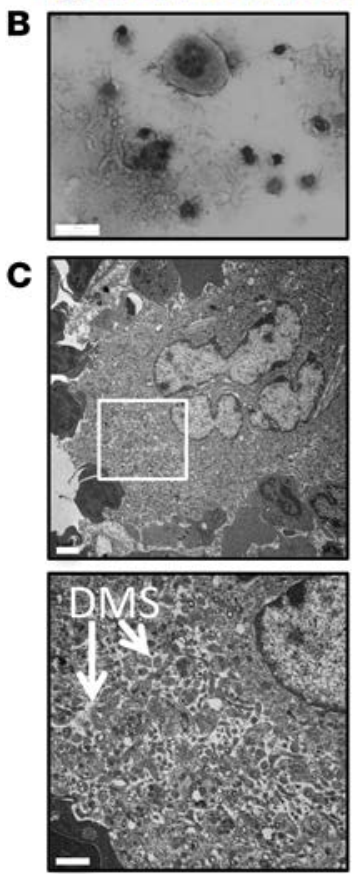

2B
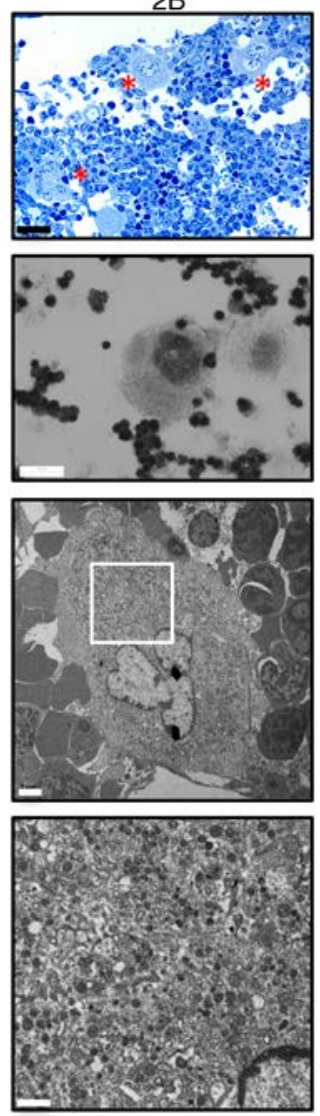

D
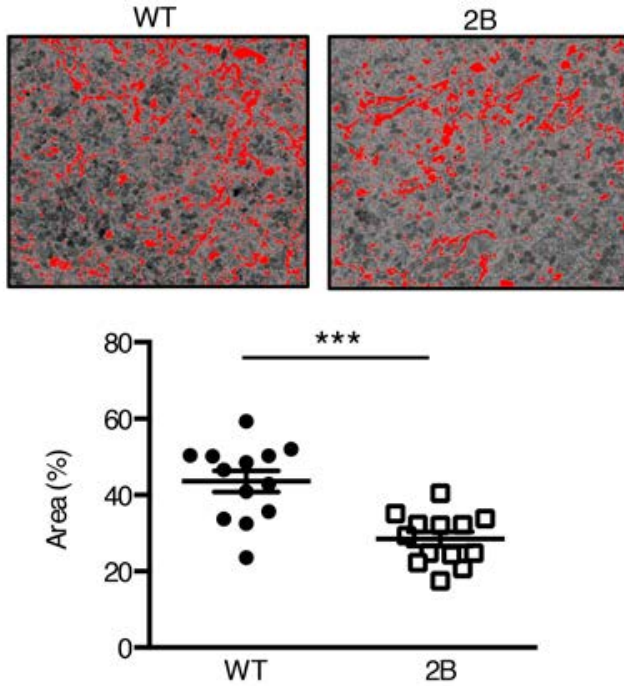

Figure 3. Type 2B mutant vWF/p.V1316M (2B) mice display abnormal megakaryocyte morphology with a decreased demarcation membrane system (DMS). (A) Representative image of the bone marrow after toluidine blue staining. Megakaryocytes (MKs) are identified with red asterisks. Scale bars: $40 \mu \mathrm{m}$. (B) Image of myelogram of the bone marrow. Bone marrow was spread on a coverslip and then stained with H\&E. Scale bars: $30 \mu \mathrm{m}$. (C) Transmission electron microscopy of stage III MKs in the bone marrow in WT and 2B mice at 2 magnifications. Scale bars: $2 \mu \mathrm{m}$ (top) and $1 \mu \mathrm{m}$ (bottom). White squares indicate the magnification zone. $n=4$. (D) Images of the threshold (Imagel software [NIH], in red) in the central zone of MKs used to quantify the area of the DMS in WT (left) and 2B MKs (right) at stage III. Quantification of the area of the DMS of 13 MKs in each group. Statistical significance was determined by Student's $t$ test. ${ }^{* *} P<0.001$.

$2 B$ mice display abnormal demarcation membrane system formation but normal MK differentiation. Because $2 \mathrm{~B}$ MKs fail to develop proplatelets, we therefore evaluated whether this defect was due to an abnormality of MK morphology and/or MK differentiation during the maturation process. In situ observation of the BM and myelogram analysis showed an increase in $2 \mathrm{~B} \mathrm{MK}$ size (Figure 3, A and B; $513 \pm 14 \mu \mathrm{m}^{2}$ vs. $435 \pm$ $12 \mu \mathrm{m}^{2}$ in $2 \mathrm{~B}$ and WT, respectively, $n=3$ mice with $50-80 \mathrm{MKs} /$ mouse, $P<0.001$ ). To further investigate MK morphology, TEM was used to visualize the demarcation membrane system (DMS), which is known to be the only source of proplatelet membranes (22) in stage III granular/mature MKs. For WT mice, TEM showed a normal MK morphology and platelet territories delimitated by a well-developed membrane system (Figure 3C). For 2B mice, the DMS was less developed within most MKs. The DMS represented $29 \% \pm 2 \%$ of the area in $2 \mathrm{~B}$ MKs, while it represented $44 \% \pm 3 \%$ in WT MKs $(P<0.001)$ (Figure 3 , C and $\mathrm{D})$. We next investigated whether this decreased development of the DMS reflected an abnormal differentiation state of the 2B MKs. As a marker of MK differentiation, we examined DNA ploidy, and we also quantified the percentage of MK/erythroid cell fraction, as well as the percentage of MK progenitors (Supplemental Figure 4) in the BM and after in vitro differentiation. No difference was observed in ploidy distribution and in progenitor populations (Supplemental Figure 5). In addition, similar numbers of MK CFU and myeloid CFU were found, indicating a normal commitment to MKs and thus a similar BM functionality of both populations (Supplemental Figure 6). Altogether, these data indicate that the p.V1316M mutation does not modify MK differentiation.

Overall, these data indicate that the macrothrombocytopenia of $2 \mathrm{~B}$ mice is unlikely to result from defects in MK differentiation, but rather suggests a defect in the formation of the DMS.

Actin disorganization and upregulation of LIMK/cofilin signaling pathway in $2 B M K$ s. Because $2 \mathrm{~B} \mathrm{MKs}$ failed to develop normal DMS and proplatelet extensions, we investigated the structure of actin, a critical component of the cytoskeleton controlling MK cytoplasmic morphogenesis (8, 23, 24). Actin distribution and organization were investigated in cultured MKs that were allowed to spread on a fibrinogen matrix. In WT MKs, cell spreading was associated with the organization of actin into lamellipodia, stress fibers, and nodules (Figure 4A). By contrast, the organization of F-actin was markedly different in 2B MKs (Figure 

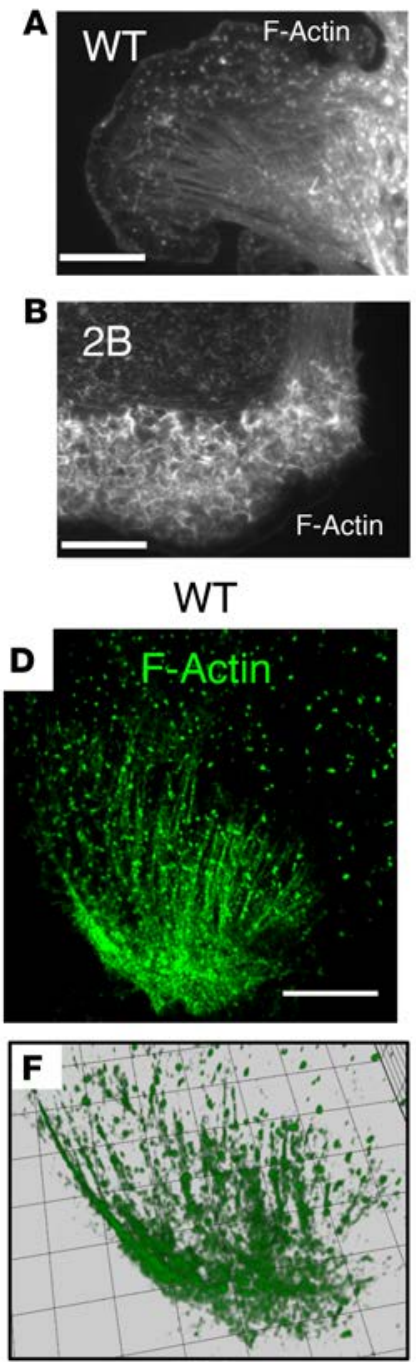

\section{C}

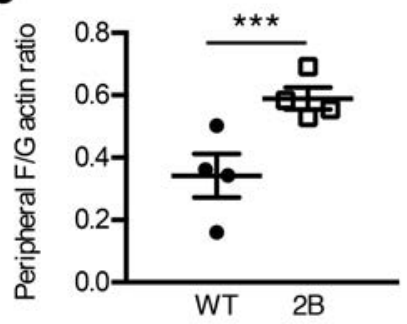

2B
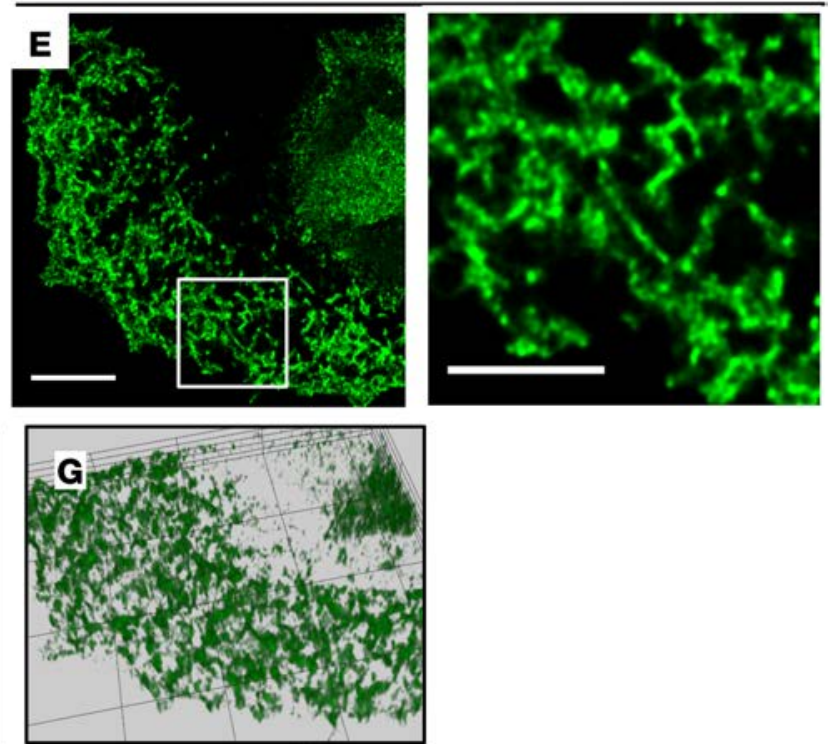

Figure 4. The actin network in type 2B mutant vWF/p.V1316M (2B) megakaryocytes (MKs). (A and B) Mature MKs after thrombopoietin-induced differentiation in culture were incubated over a fibrinogen matrix for 3 hours. Representative images of the actin structure of WT (A) and 2B MKs (B). Scale bars: $20 \mu \mathrm{m}$. (C) Quantification of the $F / G$-actin integrated intensity in the peripheral zone of 4 separate experiments. Statistical significance was determined by Student's $t$ test (20-45 MKs were analyzed/experiment). ${ }^{* *} P$ $<0.001$. (D and $\mathbf{E}$ ) Confocal microscopy images of $F$-actin structure in WT (D) and $2 B$ MKs (E). One image of the z-stack is presented. Scale bars: $10 \mu \mathrm{m}$. White squares indicate the magnification zone in 2B MKs (right). ( $\mathbf{F}$ and $\mathbf{G}$ ) The complete $z$-stacks are represented as a $3 \mathrm{D}$-volumetric reconstruction using Icy software. F, WT; G, 2B.

4, B and D-G). Although 2B MKs displayed lamellipodia, actin filaments behind the leading edge were unstructured in $66 \% \pm 2 \%$ of $2 \mathrm{~B}$ MKs compared with $7 \% \pm 4 \%$ in WT MKs $(P<0.001$; Figure 4 , A, B, and $\mathrm{D}-\mathrm{G})$. These unusually thick peripheral zones enriched in disorganized $\mathrm{F}$-actin displayed an increased ratio of F-actin to globular actin (G-actin), indicating accumulation of actin polymerization (Figure 4C and Supplemental Figure 7). Given the disorganization of the actin network, we considered the hypothesis that the activity of the actin turnover-regulating protein cofilin (8) or levels of the actin-binding scaffold protein IQGAP1 (25) could be modulated in 2B MKs. However, levels of IQGAP1 were similar in 2B and WT MKs (Figure 5A). In contrast, when analyzing levels of the inactive phosphorylated form of cofilin (at Ser3), we observed that it was increased significantly (2.6-fold, $P<0.01$ ) in 2B MKs compared with WT MKs (Figure 5A). We subsequently analyzed levels of phosphorylated LIMK, the kinase that induces cofilin phosphorylation. Interestingly, the level of phosphorylated LIMK was also increased significantly (3.9-fold, $P<0.001$ ) in 2B MKs compared with WT MKs (Figure 5A). Of note, molecules reported to be involved in the GPIba/vWF-dependent signaling pathway (such as phosphorylated Lyn, p38MAPK, and PLC $\gamma 2$ ) were not modified in 2B MKs (Supplemental Figure 8). We then investigated the localization of the total and phosphorylated forms of cofilin in MKs. In WT MKs, the distribution of both forms was homogeneous. However, in $2 \mathrm{~B} \mathrm{MKs}$, both forms were recruited to the disorganized F-actin structures, indicating accumulation of cofilin in the disturbed actin polymerization zones (Figure 5B).

Together, these data indicate defects in the actin network of 2B MKs pointing to a perturbation of the LIMK/cofilin axis.

$L I M K$ inhibition rescues actin turnover and proplatelet formation in both mouse and human $2 B M K s$. To investigate whether hyperactivity of LIMK contributed to disorganization of the actin network in 2B MKs, 
A

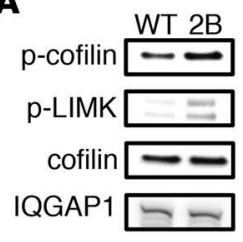

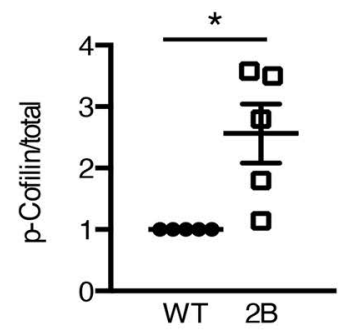

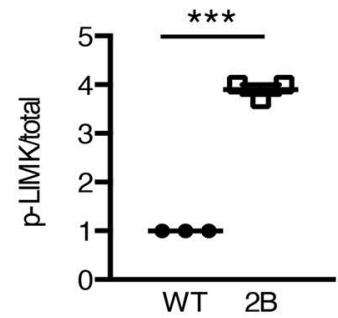

B

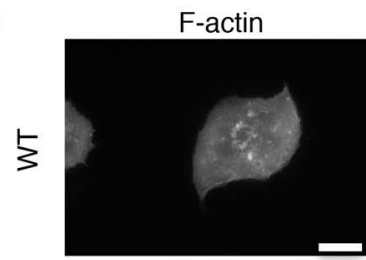

$\stackrel{m}{N}$
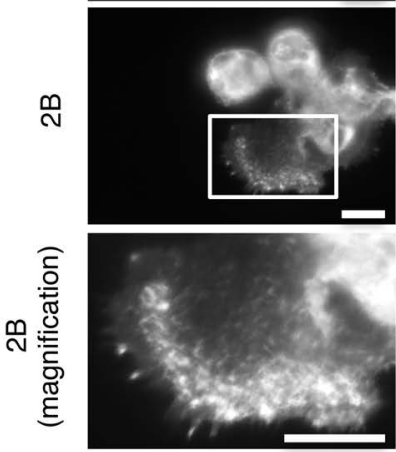
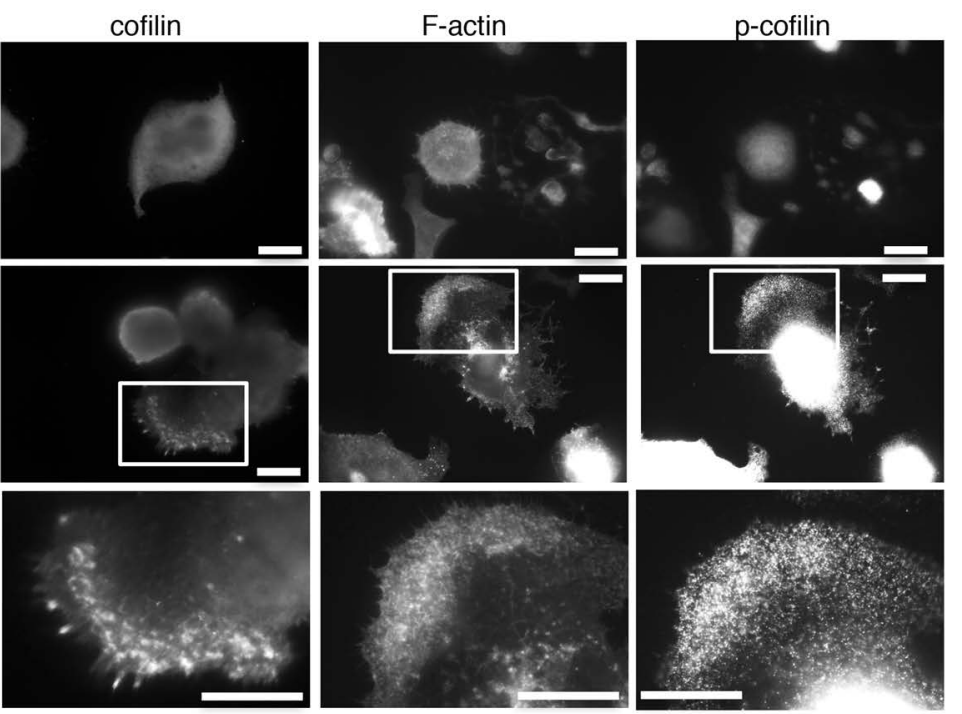
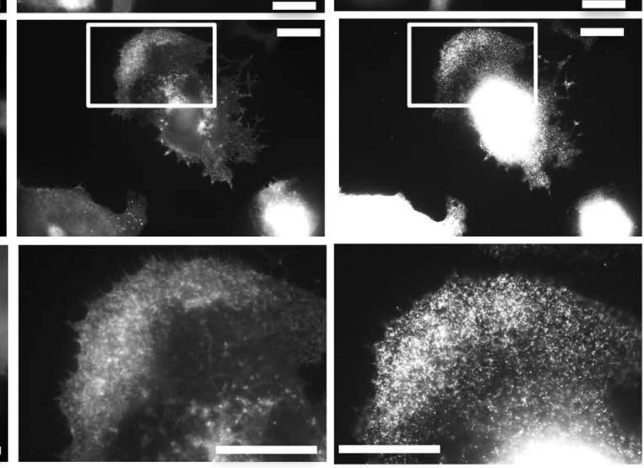

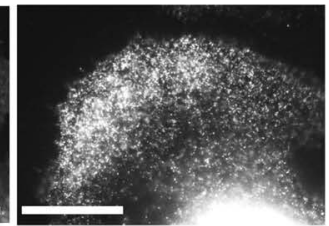

Figure 5. The LIM kinase (LIMK)/cofilin signaling pathway is dysregulated in type 2B mutant vWF/p.V1316M (2B) megakaryocytes (MKs). (A) Western blot of IQGAP1, cofilin, phospho-cofilin ( $p$-cofilin), and $p$-LIMK, and quantification of $p$-cofilin $(n=5)$ and p-LIMK $(n=3)$ in mature MKs after thrombopoietin-induced differentiation. $n=5$. (B) WT and 2B MKs after thrombopoietin-induced differentiation in culture were incubated over a fibrinogen matrix for 3 hours and stained for F-actin and total cofilin or p-cofilin (on Ser3). Scale bars: $10 \mu \mathrm{m}$. White squares indicate the magnification zone. Statistical significance was determined by Student's $t$ test. ${ }^{*} P<0.05$, ${ }^{* * *} P<0.001$

experiments were performed using a LIMK inhibitor (LIMKi). First, treatment of 2B MKs with LIMKi (10 $\mu \mathrm{M})$ strongly decreased phosphorylation of cofilin, thereby increasing cofilin activity (Figure 6A). In addition, a decreased accumulation of actin was observed: only $16 \% \pm 11 \%$ of $2 \mathrm{~B}$ MKs presented with actin accumulation after LIMKi treatment compared with $68 \% \pm 2 \%$ after vehicle treatment $(P<0.05)$ (Figure $6 \mathrm{~B}$ and Supplemental Figure 9). We next evaluated proplatelet formation on a fibrinogen matrix. For WT MKs, the percentage of proplatelet-forming MKs was similar in LIMKi- and vehicle-treated (DMSO) cells (Figure 6C), which is compatible with the normal platelet count reported in LIMK-deficient mice (26). Interestingly, while the percentage of $2 \mathrm{~B} \mathrm{MKs}$ that formed proplatelets remained low in the presence of vehicle $(18 \% \pm 1 \%)$, treatment of $2 \mathrm{~B}$ MKs with LIMKi restored proplatelet formation to a level similar to that of vehicle-treated WT MKs $(43 \% \pm 2 \%$ and $52 \% \pm 10 \%$ in LIMKi-treated $2 \mathrm{~B}$ MKs and vehicle-treated WT MKs, respectively; $P>0.05$; Figure 6C). Moreover, the average diameter of platelet-like structures occurring along the proplatelets returned to normal in LIMKi-treated $2 \mathrm{~B} \mathrm{MKs}$ but not in vehicle-treated cells $(4.1 \pm 0.5 \mu \mathrm{m}$ vs. $9.5 \pm 0.5 \mu \mathrm{m}$, respectively $[P<0.01]$ compared with $4.6 \pm 0.7 \mu \mathrm{m}$ in WT MKs) (Figure $6 \mathrm{D}$ ). These data indicate that LIMK inhibition rescues active cofilin levels and actin reorganization in 2B MKs, resulting in normal proplatelet formation in vitro.

We next investigated in human VWD-type 2B cultured MKs whether a similar dysregulation of the LIMK/cofilin/actin signaling pathway was also apparent. The patient harboring the p.V1316M mutation presented with a loss of high-molecular-weight vWF multimers and markedly reduced platelet counts (40 $\times 10^{9}$ platelets/1) at the time of examination. Human $\mathrm{CD} 34^{+}$cells from peripheral blood of control and the patient were differentiated in vitro in the presence of thrombopoietin (TPO). Following maturation, MKs were allowed to spread on a fibrinogen-coated surface in order to examine actin structure and cofilin phosphorylation. This approach revealed that actin was unstructured in the vast majority of $2 \mathrm{~B}$ MKs, with actin accumulation in peripheral zones (Figure 7A), similar to that found in murine 2B MKs. Interestingly, regions of actin accumulation were enriched in phosphorylated cofilin, the inactive form (Figure 7A), suggesting that the defect in actin turnover was linked to hyperphosphorylation of cofilin. Indeed, Western blot analysis confirmed an increase in phosphorylated cofilin compared with human control MKs (Figure 7B). We then analyzed proplatelet formation of human 2B and control MKs. At day 11, 2B MKs exhibited 

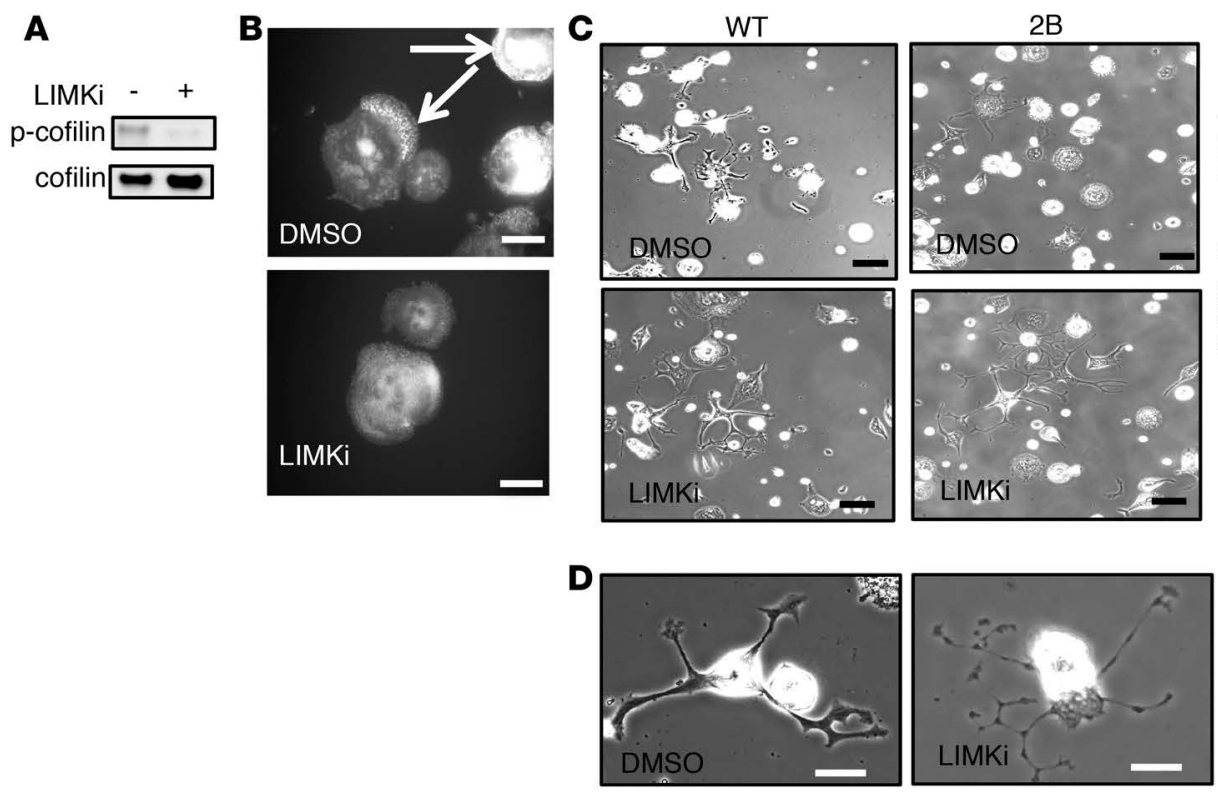

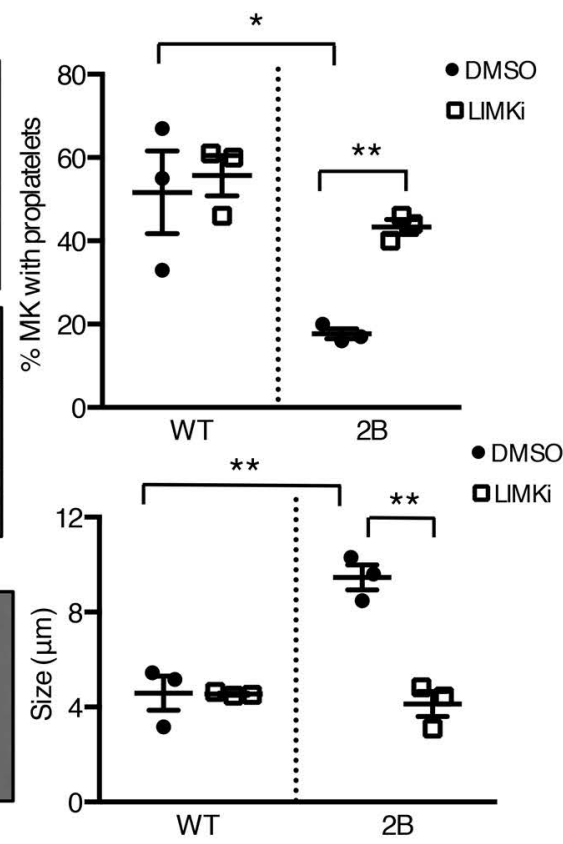

Figure 6. Inhibition of LIM kinase (LIMK) rescues proplatelet formation in type 2B mutant vWF/p.V1316M (2B) megakaryocytes (MKs). (A) Western blot of phospho-cofilin (p-cofilin) in 2B MKs in the presence or absence of LIMK inhibitor (LIMKi). Mature MKs were incubated for 3 hours with $10 \mu M$ LIMKi or DMSO (1:500 vol/vol) and then lysed in SDS denaturing buffer. $n=3$. (B) Representative images of 4 separate experiments of the actin structure (white arrows) in 2B MKs treated or not with LIMKi $(10 \mu \mathrm{M})$. DMSO was used as control. Scale bars: $20 \mu \mathrm{m}$. (C) Mature MKs after thrombopoietin-induced differentiation in culture were incubated over a fibrinogen matrix for 5 hours in the presence or absence of LIMKi (10 $\mu$ M). DMSO was used as control. Representative images of MKs forming proplatelets from 3 separate experiments. Scale bars: $50 \mu \mathrm{m}$. Quantification of the percentage of proplatelet-forming MKs in the presence or absence of LIMKi (right). The percentage of cells was measured in 3 separate experiments (50-80 MKs were analyzed/experiment). (D) Representative images (left) of 2B MKs in the presence or absence of LIMKi. Scale bars: $20 \mu \mathrm{m}$. Graph of the size of platelet-like structures (right) was measured in 3 separate experiments. Statistical significance was determined by 1-way ANOVA followed by Dunnett's test. ${ }^{*} P<0.05,{ }^{* *} P<0.01$.

a proplatelet formation defect compared with control ( $9 \%$ vs. $23 \%$ of proplatelet-forming MKs in patient and control, respectively), with the 2B MKs having shorter proplatelets and larger swellings than the control MKs (Figure 7, C and D). We then sought to rescue proplatelet formation in human 2B MKs using LIMKi. Whereas LIMKi had no effect on proplatelet formation (Figure 7, C and D) and on the average diameter of platelet-like structures in human control MKs (data not shown), LIMK inhibition restored proplatelet formation in 2B MKs ( $\sim 16 \%$ vs. $\sim 9 \%$ of proplatelet-forming MKs in LIMKi- and vehicle-treated $2 \mathrm{~B}$ MKs, respectively; Figure 7, C and D. These percentages are based on analyzing several fields of cells.). Also, we observed that proplatelet organization returned to normal, with thin and long extensions (Figure 7D) and a decreased average diameter of platelet-like structures in LIMKi-treated 2B MKs $(1.4 \mu \mathrm{m})$ compared with vehicle-treated patient MKs $(2.9 \mu \mathrm{m})$ (Figure 7E). Our results demonstrate that in both mice and humans, the LIMK/cofilin/actin signaling pathway is dysregulated in the presence of the type $2 \mathrm{~B}$ p.V1316M mutation and that LIMK inhibition restores (near) normal proplatelet formation in vitro.

RhoA signaling pathway regulates LIMK and coflin phosphorylation and restricts proplatelet formation in mouse $2 B$ MKs. Phosphorylation of LIMK and cofilin has been described to be regulated by Rho-family GTPases (reviewed in ref. 27). We then investigated whether a modified Rac1 or RhoA activity in mouse 2B MKs could be responsible for the upregulation of LIMK and cofilin. Interestingly, we found an upregulation of RhoA-GTP in mature 2B MKs compared with WT MKs (Figure 8A), but no difference was observed for Rac-GTP (data not shown). To investigate whether hyperactivity of RhoA contributed to the upregulation of LIMK and cofilin in 2B MKs, experiments were performed using a specific RhoA kinase (ROCK, effector of RhoA) inhibitor, Y-27632. First, treatment of mature WT and 2B MKs with Y-27632 $(10 \mu \mathrm{M})$ strongly decreased phosphorylation of both LIMK and cofilin, indicating that RhoA controls LIMK and cofilin phosphorylation in WT and 2B MKs (Figure 8B). We next evaluated proplatelet formation on a fibrinogen matrix. For 2B MKs, the percentage of proplatelet-forming MKs and the number of proplatelets per MK were increased in Y-27632-treated cells compared with vehicle-treated (DMSO) cells (Figure $8 \mathrm{C}$ ). Of note, WT MKs treated with the ROCK inhibitor also showed an increase in the percentage of 
A
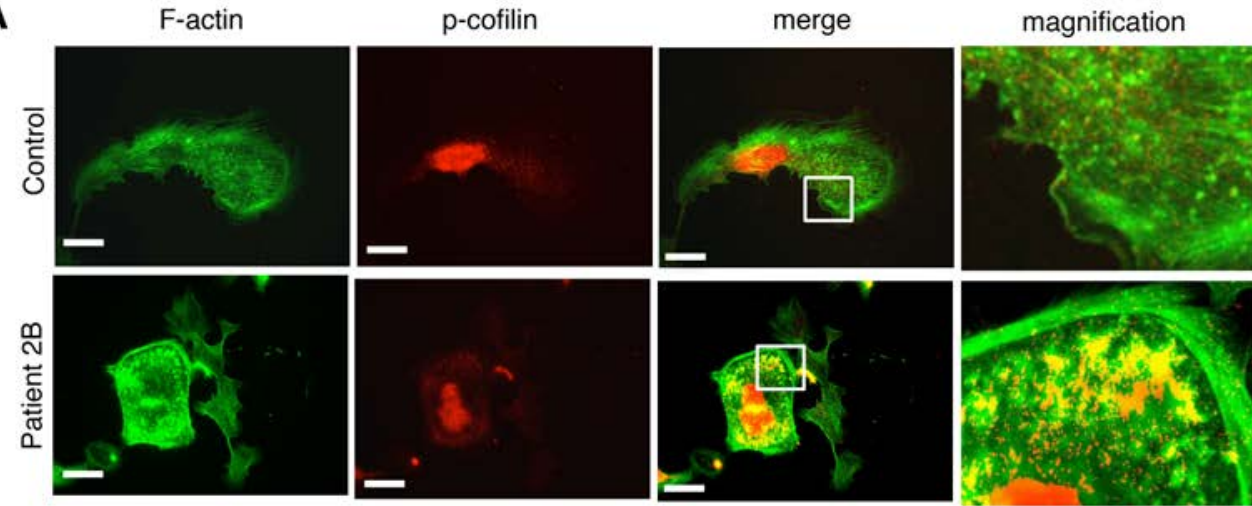

B

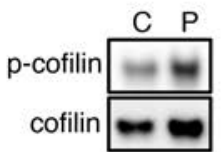

D
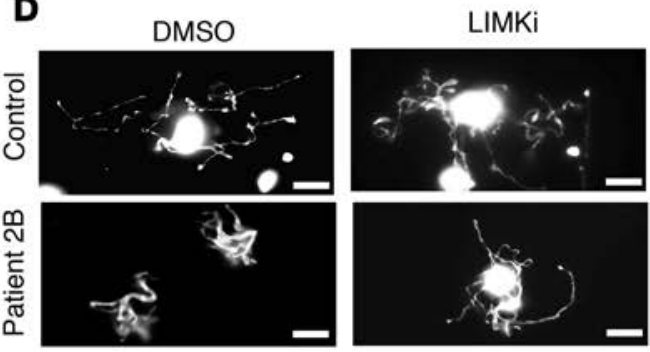

C
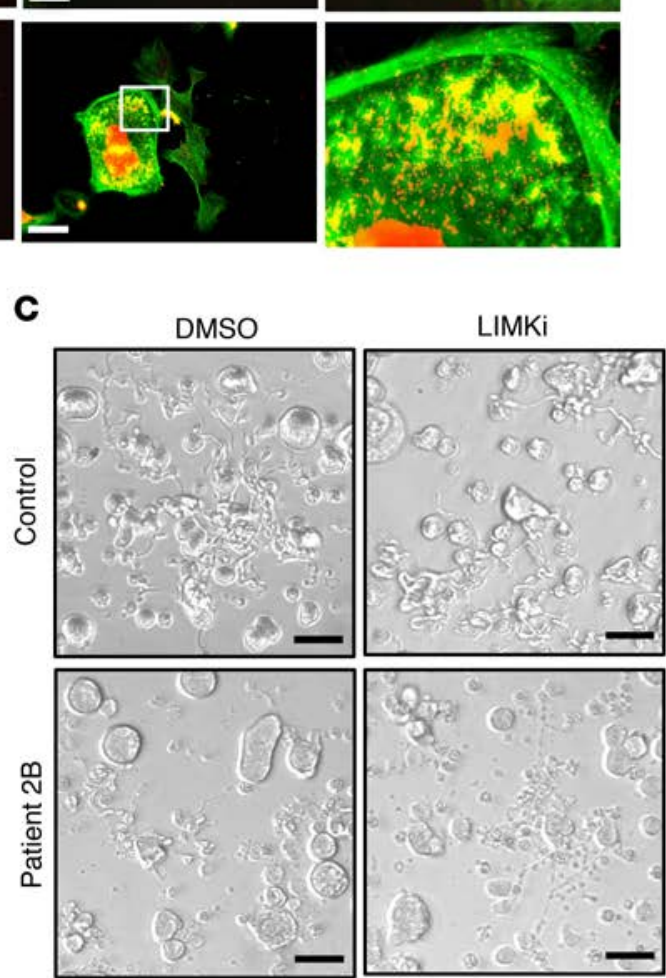

\section{E}

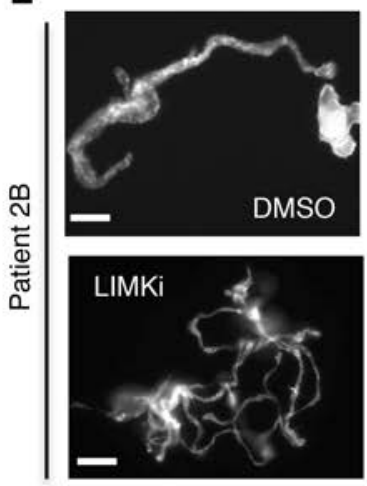

Figure 7. Human type 2B mutant vWF/p.V1316M (2B) megakaryocytes (MKs) exhibit defective actin structure and proplatelet formation, which is rescued by LIM kinase (LIMK) inhibition.

(A) Control and patient 2B MKs after thrombopoietin-induced differentiation in culture were incubated over a fibrinogen matrix for 3 hours and stained for F-actin (green) and the Ser3-phosphorylated cofilin (p-cofilin, red). Scale bars: 10 $\mu \mathrm{m}$. White squares indicate the magnification zone. (B) Western blot of $p$-cofilin and total cofilin in control (C) and in patient MKs (P). (C) Proplatelet formation in liquid culture in the presence or the absence of LIMKi at day 11, in control and in patient MKs. LIMKi $(10 \mu \mathrm{M})$ was added at day 10 ; DMSO (0.2\%) was used as vehicle. Scale bars: $50 \mu \mathrm{m}$. (D) Control and patient 2B MKs after thrombopoietin-induced differentiation in culture were spread over a polylysine-coated coverslip for 3 hours and stained for F-actin. Scale bars: $20 \mu \mathrm{m}$. (E) Proplatelet structure after F-actin staining and graph of the size of platelet-like structures in the $2 \mathrm{~B}$ patient in the presence or absence of LIMKi. Scale bars: $5 \mu \mathrm{m}$. MKs in the presence of DMSO $(n=15)$ and MKs in the presence of LIMKi $(n=10)$ were analyzed for the size of platelet-like structures. Statistical significance was determined by Student's $t$ test. ${ }^{* *} P<0.001$.

proplatelet-forming cells (Figure 8C), in agreement with previous observations (28). Taken together, our data demonstrate that the increase in LIMK and cofilin phosphorylation in 2B MKs is due to upregulation of the RhoA signaling pathway restricting proplatelet formation.

In vivo administration of LIMKi increases platelet counts and reduces $M P V$ in $2 B$ mice. Because our in vitro data demonstrate that LIMK inhibition was able to rescue proplatelet formation in $2 \mathrm{~B} \mathrm{MKs}$, we then investigated the effect of LIMKi in vivo. LIMKi or vehicle was injected subcutaneously daily for 3 days in WT and $2 \mathrm{~B}$ mice. Hematologic parameters were measured before administration and then at days 6,8 , and 10. Whereas LIMKi did not affect platelet count (Figure 9A) and MPV (data not shown) in WT mice, it increased the platelet count in 2B mice at days 6 and 8 compared with vehicle (Figure 9B). For instance, platelet count determined at day 6 was $706 \pm 44 \times 10^{3}$ platelets/ $\mu 1$ in LIMKi-treated $2 \mathrm{~B}$ mice versus 483 $\pm 50 \times 10^{3}$ platelets $/ \mu 1$ in vehicle-treated $2 \mathrm{~B}$ mice $(P<0.001)$, representing an increase of $64 \%$ versus $10 \%$ 
A

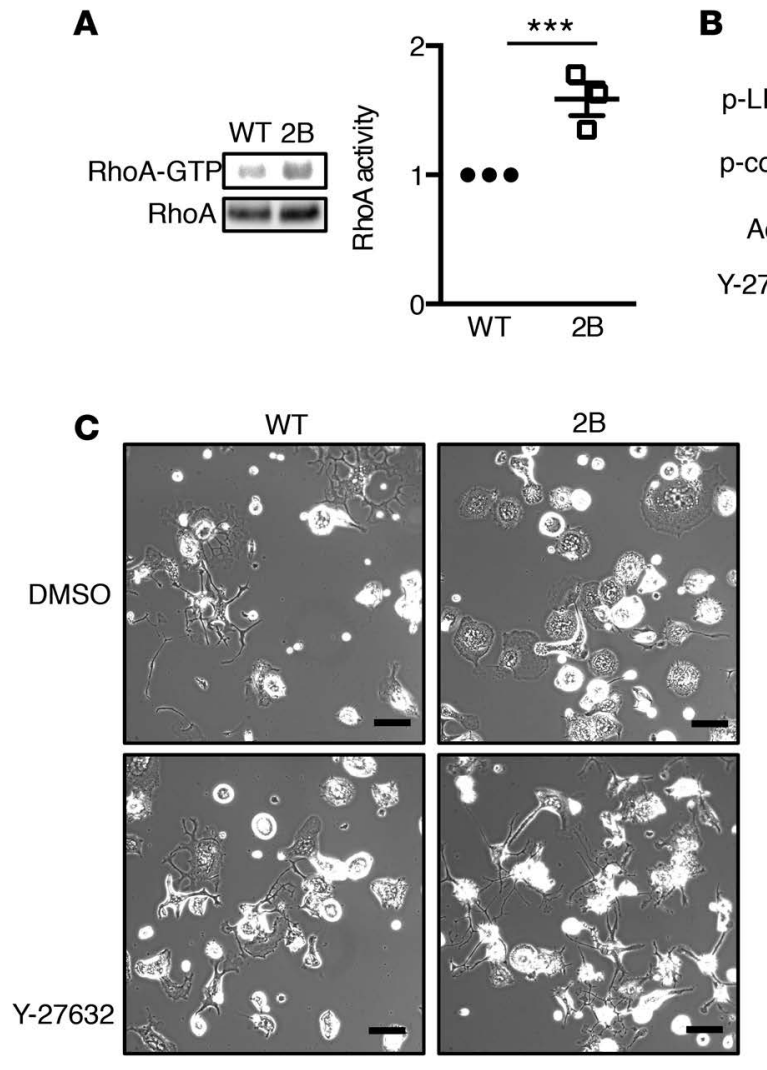

B

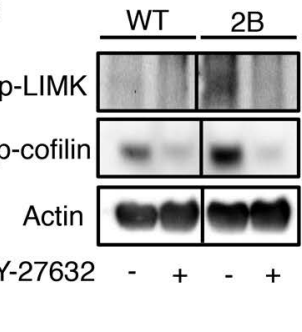

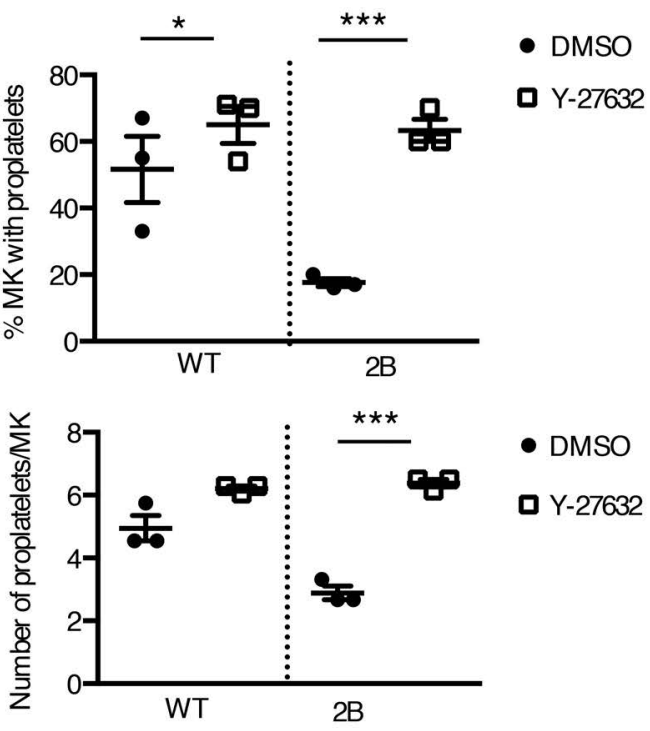

Figure 8. Active RhoA is upregulated in type 2B mutant vWF/p.V1316M (2B) megakaryocytes (MKs) and controls the phosphorylation of LIM kinase (LIMK) and cofilin. (A) Pull-down assays of RhoA-GTP were performed in WT and 2B mature MKs. Western blot and quantification of RhoA and Rhoa-GTP. Statistical significance was determined by Student's $t$ test. ${ }^{* *} P<0.001, n=3$. (B) Western blot of LIMK and cofilin phosphorylation in WT and 2B MKs in the presence or the absence of the RhoA kinase (ROCK) inhibitor, Y-27632. Mature MKs were incubated for 3 hours with $\mathrm{Y}-27632(10 \mu \mathrm{M})$ or DMSO $(0.2 \%)$ and then lysed in SDS denaturing buffer. $n=3$. These samples were run in the same gel but the lanes were noncontiguous, as indicated by the separate boxes. (C) Mature MKs after thrombopoietin-induced differentiation in culture were incubated over a fibrinogen matrix for 5 hours in the presence or absence of the ROCK inhibitor $(10 \mu \mathrm{M})$ or DMSO $(0.2 \%)$ used as control. Representative images of MKs forming proplatelets. Scale bars: $50 \mu \mathrm{m}$. Quantification of the percentage of proplatelet-forming MKs (top graph) and the number of proplatelets/MK (bottom graph) in 3 separate experiments. Statistical significance was determined by 1-way ANOVA followed by Dunnett's test (50-75 MKs were analyzed/experiment). ${ }^{*} P<0.05,{ }^{* * *} P<0.001$.

in LIMKi- and vehicle-treated 2B mice, respectively. Importantly, LIMKi treatment decreased MPV (at day 6 the MPV was $7.4 \pm 0.2 \mathrm{fl}$ in LIMKi-treated 2B mice vs. $8.1 \pm 0.1 \mathrm{fl}$ in vehicle-treated 2B mice, $P<$ 0.01 ) (Figure 9C). Of note, vehicle treatment did not significantly change the platelet counts and MPV at the indicated time points compared with untreated mice. Other blood parameters such as red and white blood cell counts, hemoglobin, and hematocrit were unmodified after the injection of the LIMKi (data not shown), suggesting a specific effect on MKs. These data demonstrate that treatment with LIMKi in 2B mice boosts platelet production and significantly reduces MPV in vivo.

We then evaluated the effects of LIMKi on the function of platelets from $2 \mathrm{~B}$ mice. In vivo, WT and 2B mice were injected subcutaneously with LIMKi $(30 \mathrm{mg} / \mathrm{kg}$ ) or DMSO as control, daily for 3 days. We then evaluated the platelet function in a tail bleeding time model at day 6 , corresponding to the maximum elevation of platelet count observed after LIMKi treatment in 2B mice (Figure 9D). The LIMKi treatment in WT mice did not modify the bleeding time. However, in 2B mice, the hemorrhagic phenotype of these mice was not restored, suggesting that elevation of platelet count was not sufficient to restore a normal hemostasis and that platelet function defects were still present. In vitro, we evaluated the effect of LIMKi on integrin $\alpha \mathrm{IIb} \beta 3$ activation. We first evaluated the integrin $\alpha \mathrm{IIb}$ expression (CD41), and observed an increase of $42 \%$ (Figure 9E). This increase correlates with the presence of giant platelets in our model, 

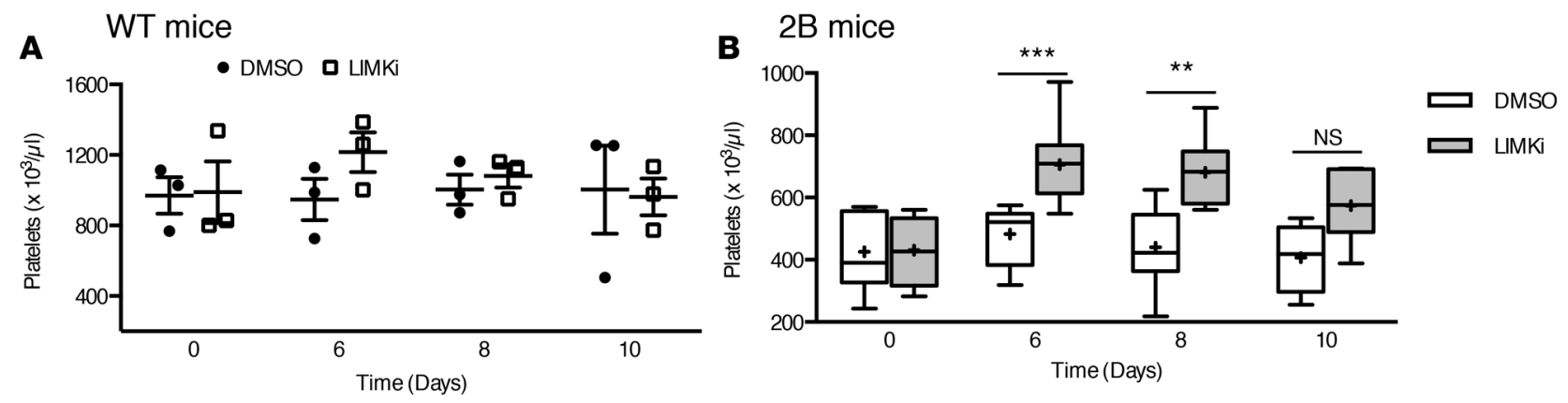

C $2 \mathrm{~B}$ mice

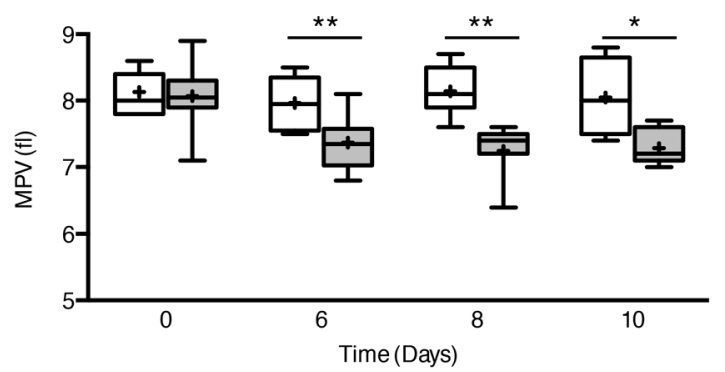

D

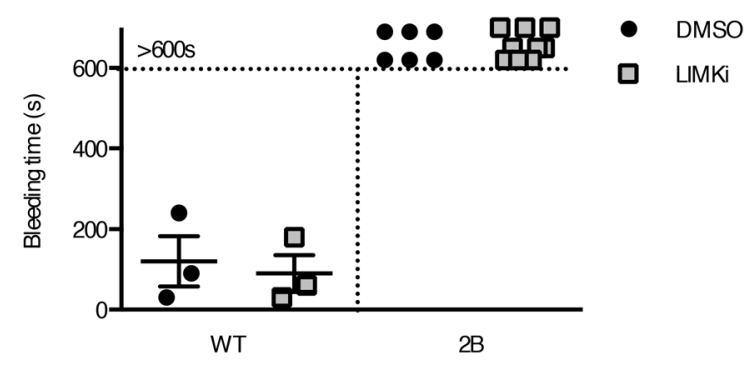

E

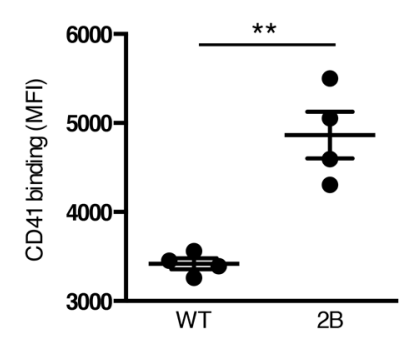

$\mathbf{F}$

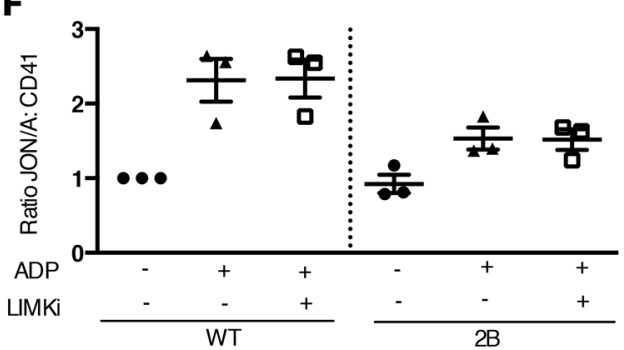

Figure 9. In vivo administration of LIM kinase inhibitor (LIMKi) increases the platelet count and reduces the mean platelet volume in type 2B mutant vWF/p.V1316M (2B) mice but does not reverse the hemostatic defect. Whole-blood platelet counts in WT (A) and 2B mice (B) of DMSO-treated and LIMKi-treated mice before and after 3 injections of LIMKi (30 mg/kg) or DMSO (1.6 $\mu \mathrm{l} / \mathrm{g}$ body weight) at various times. $n=3$. Mean platelet volume (MPV) (C) of DMSO-treated 2B mice and LIMKi-treated 2B mice. $n=4-12$ mice in each group. Statistical significance was determined by 1-way ANOVA followed by Dunnett's test. ${ }^{*} P<0.05$, ${ }^{* *} P<0.01$, ${ }^{* *} P<0.001$. NS, not significant. (D) Tail bleeding time in WT $(n=3)$ and $2 \mathrm{~B}$ mice $(n=6$ and 9$)$ of DMSO-treated mice and LIMKi-treated mice after 3 injections of LIMKi $(30 \mathrm{mg} / \mathrm{kg})$ or DMSO. Integrin $\alpha$ llb $\beta 3$ expression $(n=4)(\mathbf{E})$ and activation $(n=3)(\mathbf{F})$ were assessed by flow cytometry using a CD41 antibody and integrin $\alpha \mathrm{llb} \beta 3 \mathrm{mAb}$ (JON/A) specific for the activated conformation of the mouse integrin, respectively. The level of activated integrin is indicated by the mean fluorescence intensity (MFI) ratio JON/A:CD41, calculated with the WT JON/A:CD41 ratio equal to 1.

with a platelet size increase of $44 \%$. Then, platelets of WT and 2B mice were incubated with LIMKi or DMSO for 10 minutes before activation with ADP as agonist. We evaluated the activation level of integrin $\alpha \operatorname{IIb} \beta 3$ by use of JON/A, a mAb specific for the activated conformation of mouse integrin $\alpha \operatorname{IIb} \beta 3$. Using flow cytometry, the level of JON/A binding to integrin $\alpha \mathrm{IIb} \beta 3$ after stimulation with ADP was slightly increased but remained lower in 2B platelets than in activated WT platelets (Figure 9F). After LIMK inhibition, the activation of integrin $\alpha \mathrm{IIb} \beta 3$ of WT platelets was not modified, and in 2B mice, the LIMKi was not able to restore the level of $\alpha \mathrm{IIb} \beta 3$ activation to that observed in WT platelets (Figure 9F). Altogether, these data show that LIMKi does not reverse the platelet function defects in vivo (measured by the tail bleeding time) and in vitro (measured by the activation of integrin $\alpha \operatorname{IIb} \beta 3$ ).

\section{Discussion}

Impaired platelet production is an important clinical problem, as it may result in severe bleeding, and many MK signaling pathways that are affected in disorders characterized by macrothrombocytopenia remain elusive. An example hereof is the bleeding disorder in VWD-type 2B, in which thrombopathy (29) and macrothrombocytopenia are part of complex clinical manifestations observed in patients (13). Long considered a paradoxical bleeding disorder because gain-of-function mutations of plasma vWF should 


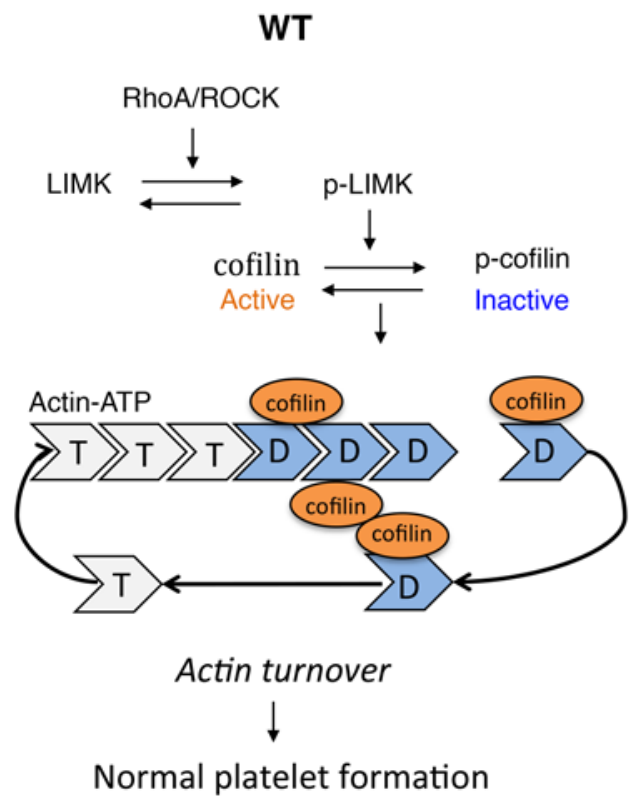

vWD-type 2B p.V1316M

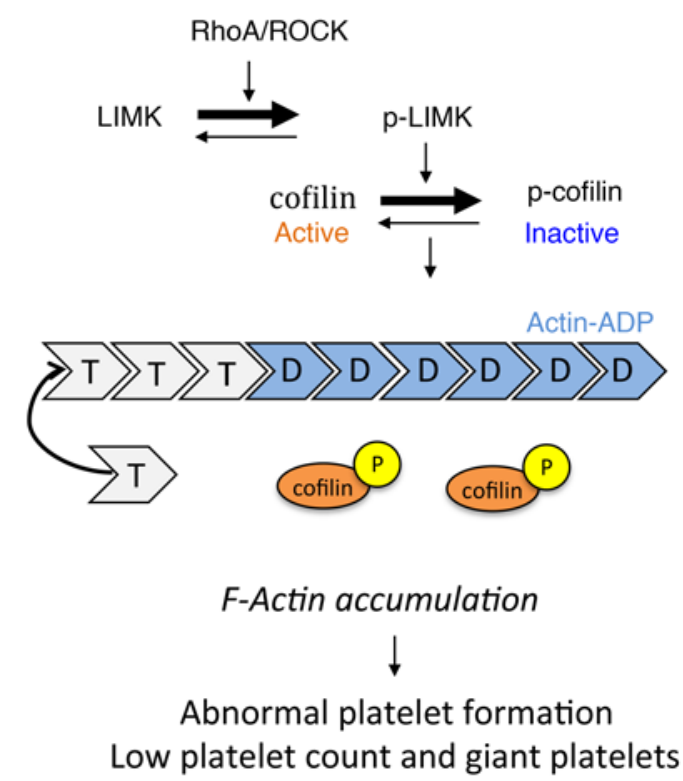

Figure 10. Scheme of the regulation of actin turnover by LIM kinase (LIMK)/cofilin in WT and type 2B mutant vWF/p.V1316M (2B) megakaryocytes (MKs). LIMKs influence the architecture of the actin cytoskeleton by regulating the activity of the cofilin family proteins. In WT mice, the equilibrium between nonphosphorylated and phosphorylated cofilin stimulates severance and depolymerization of actin, and promotes actin filament turnover, leading to proper platelet production. By contrast, in 2B MKs, the inactivation of cofilin by phosphorylation at Ser3 is upregulated. This leads to cofilin losing its ability to bind actin and then suppresses actin turnover (2). RhoA/ ROCK regulates the LIMK/cofilin pathway in WT MKs and is upregulated in 2B MKs. ROCK, RhoA kinase.

have given rise to a prothrombotic condition, it is now accepted that several causes underlie the thrombocytopenia, such as an increased platelet clearance of vWF/platelet complexes (10). However, the presence of giant platelets also point to vWF-type $2 \mathrm{~B}$ mutants playing a role in the fine regulation of the processes that result in platelet production. Indeed, previous studies have reported that proplatelet formation is disturbed in VWD-type 2B $(14,30)$, an observation that we were able to reproduce in 2B mice (Figure 2). The severity of VWD-type $2 \mathrm{~B}$ is strikingly mutation dependent and one of the most severe mutations is the p.V1316M replacement, which is associated with constant thrombocytopenia and the presence of giant platelets (15). We therefore used our genetically engineered 2B mice to investigate in more detail the different steps that lead to the formation of platelets in order to determine the mechanism of this macrothrombocytopenia. This analysis first revealed that the $2 \mathrm{~B}$ mice were indistinguishable from WT mice in all steps of differentiation that are required for the generation of mature round-shaped MKs (Supplemental Figure 5). This seems compatible with the notion that $\mathrm{vWF}$ expression is initiated at the pre-MK stage $(31,32)$. Thus, vWF is absent in early stages of megakaryocytopoiesis and mutant vWF/p.V1316M will therefore not influence these parts of the process.

However, mature MKs from the 2B mice did display a number of important differences compared with those in WT mice. First, we observed that 2B MKs were significantly increased in size (approximately 20\%) compared with WT MKs (Figure 3). More importantly, DMS, which is the only known source of proplatelet membranes (22) and originates in part from plasma membrane invagination (33), was strongly reduced (Figure 3). Given that all markers of MK maturity (such as CD41, CD42b, and the TPO receptor) were present, this underdeveloped DMS seems to be the consequence of a reduced capacity to generate these membranes. Consecutive stages of DMS organization do rely on F-actin dynamics to polarize membranes (24) and are also controlled by ROCK (34). It seems thus conceivable that the insufficiently developed membrane reservoir is in part responsible for the reduced platelet count and/or the increased platelet size in these $2 \mathrm{~B}$ mice through dysregulation of $\mathrm{F}$-actin dynamics and RhoA activity, both found to be involved in proplatelet formation defects in $2 \mathrm{~B}$ MKs.

A second aberrant characteristic that could affect platelet count and/or platelet size in 2B MKs relates 
to the defect in actin cytoskeleton organization. Indeed, microscopic analysis revealed that actin behind the leading edge is unstructured in the peripheral zone of $2 \mathrm{~B} \mathrm{MKs}$, with an accumulation of actin filaments. Changes in actin polymerization are key to many processes involving the formation of proplatelets (4-6, 8, 35). In particular, the formation of extensions and proplatelet shaft bifurcations are clearly actin dependent, and it is not surprising that actin plays a fundamental role in the terminal step of proplatelet production (8). Recently, it has been described that proplatelet formation was impaired and MKs displayed a reduced number of proplatelet tips of altered diameter, while actin polymerization was increased in vitro (36).

The signaling cascade that controls actin dynamics involves a variety of proteins, including cofilin. When nonphosphorylated, cofilin stimulates severing and depolymerization of actin and promotes actin turnover. In contrast, when phosphorylated at Ser3, cofilin enters an inactive state and loses its ability to bind actin (reviewed in ref. 2). Consequently, actin filaments are stabilized and accumulate in areas enriched in phosphorylated cofilin (Figures 5 and 7). This extends previous findings showing that hyperphosphorylation of cofilin induces a platelet-formation defect $(8,35)$. In addition, mutations in the cofilin partner Aip1/Wdr1 lead to macrothrombocytopenia (37). We investigated whether dysregulation of cofilin could explain the defect in the control of the actin network in 2B MKs. Analysis of both human and murine 2B MKs identified the presence of significantly increased levels of phosphorylated cofilin, i.e., the inactive form of cofilin. Our data sustain the following model: in 2B mice, the balance between the nonphosphorylated and phosphorylated cofilin is disturbed and cofilin loses its ability to bind actin, which suppresses actin turnover (Figure 10).

Because LIMK participates in actin dynamics by phosphorylating cofilin (1), additional investigative work was then directed to LIMK. Members of the LIMK family (Lin-11/Isl-1/Mec-3 domain-containing protein kinases) contain $2 \mathrm{~N}$-terminal LIM domains that are protein-binding domains frequently found in cytosolic proteins interacting with the actin cytoskeleton. Indeed, LIMKs have been shown to influence the architecture of the actin cytoskeleton by regulating the activity of the cofilin family proteins (2). We also found increased levels of phosphorylated LIMK in 2B MKs (Figure 5), which were probably responsible for increased levels of inactive cofilin, thereby dysregulating actin turnover. To support this hypothesis, we performed in vitro and in vivo studies using a LIMKi. When added to $2 \mathrm{~B}$ MKs, the LIMKi resulted in a reduction of the level of the inactive, phosphorylated form of cofilin (Figure 6). Furthermore, the inhibitor normalized not only formation of proplatelet-forming MKs, but also the size of the new proplatelets (Figures 6 and 7). Also, treatment of mice with the LIMKi had tremendous implications, as it resulted in significantly increased platelet counts and reduced MPV values (Figure 9). These in vitro and in vivo data confirm that dysregulation of the LIMK/cofilin pathway in VWD-type 2B is at the origin of impaired proplatelet formation, and thereby contributes to the macrothrombocytopenic phenotype of the patients (Figure 10).

How does the vWF/p.V1316M mutation induce dysregulation of the LIMK/cofilin pathway? Two aspects are of relevance in this regard. First, it has been reported that artificial, ristocetin-stimulated complex formation between vWF and GPIb $\alpha$ induces activation of the LIMK/cofilin pathway (26). We therefore considered the possibility that outside-in signaling induced upon complex formation by freshly secreted vWF/p.V1316M and cell-surface-exposed GPIb $\alpha$ could be responsible for the activation of the LIMK/ cofilin pathway. However, in line with previous reports (38), we could not detect any vWF at the MK surface, by immunofluorescence or by flow cytometry. This suggests that the mutation p.V1316M enables vWF to modulate the signaling cascade from its endogenous location, as previously described in human 2B MKs (30). Indeed, addition of $2 \mathrm{~B} v \mathrm{vW}$ in the cell culture boosts platelet production and has an effect opposite that of vWF synthesized by the MK (30).

The second aspect that is of relevance is how vWF/p.V1316M might be able to disturb the LIMK/ cofilin pathway. It is known that the activity of LIMK is regulated by the Rho-family GTPases RhoA, Rac1, and Cdc42 via their effector kinases ROCK and p21-activated kinase (reviewed in ref. 27). Several of these components have indeed been reported to be involved in the regulation of the actin network and proplatelet formation $(28,35)$. In 2B MKs, an increase in the active form of RhoA (RhoA-GTP) but not Rac1 was observed. Moreover, the presence of ROCK inhibitor reduces the level of phosphorylated LIMK and of inactive cofilin, both in WT and 2B MKs (Figure 8) and normalized the formation of proplatelet-forming 2B MKs. Our data are in agreement with previous studies providing evidence that the Rho/ROCK pathway acts as a negative regulator of proplatelet formation $(28,39)$. Indeed, overexpression of a spontaneously active RhoA leads to a decrease in proplatelets, indicating that RhoA activation inhibits proplatelet formation (28). Of note, it was reported that the Rho signaling pathway could be 
controlled by the small GTPase Rap1 (40). Interestingly, we have recently demonstrated in VWD-type 2B p.V1316M that the thrombopathy originates from an impaired activation of Rap1 (29). It is therefore tempting to speculate that a similar lack of Rap1 activation occurs in 2B MKs, thereby dysregulating the RhoA/ROCK/LIMK/cofilin pathway, which subsequently provokes destabilization of the actin network. This is ultimately manifested by a severely affected proplatelet formation, and explains at least in part the macrothrombocytopenia in VWD-type $2 \mathrm{~B}$.

The risk of bleeding in VWD-type 2B patients with thrombocytopenia was shown to be 5 times higher than that of patients with a normal platelet count, notably with the severe p.V1316M mutation (13). Treatment of VWD relies either on replacement therapy with vWF concentrates or on desmopressin (DDAVP) to increase levels of circulating vWF (41). However, DDAVP is not recommended in VWD-type 2B because of the possibility of worsening the thrombocytopenia and further increasing the risk of bleeding (42). Given these major challenges in the prevention or treatment of bleeding in VWD-type 2B patients with thrombocytopenia, a breakthrough might be provided by a treatment favoring platelet production. Whether LIMK could also represent a potential therapeutic target in the treatment of VWD-type 2B was tested in our study. LIMK inhibitors are already under consideration for several indications (43), such as cancer (44), elevated intraocular pressure (45), pulmonary hypertension (46), and human immunodeficiency virus type 1 (HIV-1) (47). Our results (Figure 9) show indeed that targeting LIMK restores platelet counts in $2 \mathrm{~B}$ mice. However, we also demonstrated that the hemorrhagic phenotype of these mice was not corrected, suggesting that elevation of platelet count was not sufficient to restore a normal hemostasis. Furthermore, we previously demonstrated that platelets from 2B patients (29) and 2B mice (17) are defective in integrin $\alpha \operatorname{IIb} \beta 3$ activation. After LIMK inhibition, the activation of integrin $\alpha \operatorname{IIb} \beta 3$ of $2 \mathrm{~B}$ platelets was not restored. Altogether, these data show that the LIMKi does not reverse the platelet function defects in vivo and in vitro and provide evidence that: (a) the platelet function defect in this model is a more important contributor to the hemostatic defect than the thrombocytopenia, and (b) the LIMK signaling pathway is not involved in the integrin $\alpha \operatorname{Ilb} \beta 3$ activation defect in severe VWD-type $2 \mathrm{~B}$. Based on the present study, LIMK inhibitors may be used to restore platelet counts in the treatment of VWD-type $2 \mathrm{~B}$ patients exhibiting thrombocytopenia, but such treatments need to be combined with hemostatic agents.

The presence of giant platelets associated with a low platelet count is a condition that occurs spontaneously in patients with the most severe VWD-type $2 \mathrm{~B}$ mutations or in stress conditions for patients with milder mutations. Because macrothrombocytopenic phenotypes are heterogeneous in VWD-type $2 \mathrm{~B}$, we can speculate that all ranges of responses could be observed, notably the impaired actin turnover related to the RhoA/LIMK/cofilin pathway. Further investigations are required to determine the involvement of this pathway in a mutation-dependent manner. By comparison, platelet-type VWD (PT-VWD) is an autosomal dominant inherited bleeding disorder caused by gain-of-function mutations of GPIb $\alpha$ conferring to platelet integrin GPIb an enhanced affinity for vWF. This disorder is associated with moderate macrothrombocytopenia, which is currently explained by the formation of vWF-platelet complexes that are then rapidly cleared from the circulation. No studies, however, have shown enhanced platelet clearance in patients with PT-VWD and defective platelet formation. Based on these premises, it is conceivable that defective proplatelet formation may also contribute to macrothrombocytopenia in PT-VWD, but this point requires further investigation. Gain-of-function mutations either in GPIb (PT-VWD) or in vWF (VWD-type 2B) lead to macrothrombocytopenia. It is therefore tempting to speculate that gain-of-function mutations in the receptor or ligand induce a common signaling pathway that dysregulates platelet production.

\section{Methods}

Additional description of materials, including a list of antibodies used, and experimental procedures can be found in the online supplemental methods and data.

Mice and hematologic analysis. The VWD-type 2B knockin mouse model (p.V1316M) has been described elsewhere (17). Mice homozygous for the p.V1316M mutation are referred to as $2 \mathrm{~B}$ mice and their control littermates as WT mice. Blood counts were determined with an automatic cell counter (Scil Vet ABC Plus, Horiba Medical).

Preparation of MKs and culture. BM cells were obtained by flushing femurs and tibiae of 2B mice and control littermates, and cells expressing 1 or more of the lineage-specific markers on their surface (Ter119, B220, CD4, CD5, CD11b, and Gr-1) were depleted using immunomagnetic beads (sheep anti-rat IgG Dynabeads, Thermo Fisher Scientific). The remaining population (referred as $\mathrm{Lin}^{-}$cells) was cultured at 
$37^{\circ} \mathrm{C}$ in $5 \% \mathrm{CO}_{2}$ in Iscove Modified Dulbecco's Medium (IMDM; Invitrogen) supplemented with $15 \%$ BIT 9500 serum substitute (STEMCELL Technologies), $\alpha$-monothioglycerol (Sigma-Aldrich), and liposomes (phosphatidylcholine, cholesterol, and oleic acid; all from Sigma-Aldrich) (48), and in the presence of 10 $\mathrm{nM}$ recombinant human TPO (Miltenyi Biotec). After 5 days of culture, the cell population was enriched in mature MKs using a $1.5 \% / 3 \%$ BSA gradient under gravity $(1 \mathrm{~g})$ for 45 minutes at room temperature as described (49). For human cells, CD $34^{+}$cells were isolated from peripheral blood by an immunomagnetic technique (Miltenyi Biotec). Human recombinant stem cell factor (SCF, $20 \mathrm{ng} / \mathrm{ml}$, Miltenyi Biotec) and human TPO $(50 \mathrm{nM})$ were added once on day 0 to the culture medium, followed by $20 \mathrm{nM}$ TPO alone on day 6 without any addition of SCF thereafter until day 12 .

Actin structure and proplatelet formation over fibrinogen matrix. Coverslips were coated with fibrinogen $\left(100 \mu \mathrm{g} / \mathrm{ml}, \mathrm{HYPHEN}\right.$ BioMed SAS) overnight at $4^{\circ} \mathrm{C}$. After washing twice with PBS, the coverslips were blocked with BSA $(5 \mathrm{mg} / \mathrm{ml})$ for 1 hour at room temperature, and washed twice with PBS before use.

For actin assay, mature MKs were plated on a fibrinogen-coated surface for 3 hours at $37^{\circ} \mathrm{C}$ and then fixed (4\% paraformaldehyde) and permeabilized with Triton X-100 (0.1\%), and actin fibers were stained with $4 \mu \mathrm{g} / \mathrm{ml}$ Alexa Fluor 488-labeled phalloidin (Thermo Fisher Scientific) and then visualized by epifluorescence microscopy (Eclipse 600, Nikon) or confocal microscopy (LSM700 confocal microscope, Carl Zeiss). Z-stack images (z-depth, $0.34 \mu \mathrm{m}$ ) were deconvolved using Huygens software (Scientific Volume Imaging). The complete z-stacks were represented as a 3D-volumetric reconstruction using Icy software (Pasteur Institute). To determine the F/G-actin ratio, MKs were stained for 30 minutes at room temperature with Alexa Fluor 488-phalloidin $(4 \mu \mathrm{g} / \mathrm{ml})$ to stain F-actin and with Alexa Fluor 594-labeled DNase I (17 $\mu \mathrm{g} / \mathrm{ml}$, Thermo Fisher Scientific) to stain monomeric G-actin. The F/G-actin integrated intensity was calculated in the peripheral zone of MKs with Fiji software (50).

For proplatelet formation assays, mature MKs were plated on a fibrinogen-coated surface for 5 hours at $37^{\circ} \mathrm{C}$. Adherent MKs were fixed ( $4 \%$ paraformaldehyde) and then visualized by TEM (see supplemental methods for details).

Patient $2 B$. The patient with a vWF mutation associated with VWD-type $2 \mathrm{~B}$ was enrolled in this study after informed consent in accordance with the Declaration of Helsinki. The patient is a man exhibiting a mutation corresponding to a p.V1316M substitution. The bleeding score of the patient was high (score 27 vs. control < 5) (12) associated with a loss of high-molecular-weight vWF multimers and a low platelet count $\left(40 \times 10^{9}\right.$ platelets $\left./ 1\right)$ at the time of examination.

Study approval. For mouse studies, housing and experiments were done as recommended by French regulations and the experimental guidelines of the European Community. This project was approved by the local ethics committee CEEA 34 under the number CEEA34.SC.040.12.

For human studies, control and patient gave written informed consent before participating in this study and were enrolled in the study in accordance with the Declaration of Helsinki. The study was approved by the Ethical Committee of the Institut National de la Santé et de la Recherche Médicale (INSERM RBM) 01-14.

Statistics. Quantitative data graphs are presented as dot plots, with the mean \pm SEM, or as box and whisker plots, with the lower hinge representing the 25 th percentile, and the upper hinge the 75th percentile. The line inside the box marks the median and the mean is represented by a cross $(+)$. The maxima and minima are at the ends of the whiskers.

Statistical analyses were performed using Prism 6 for Mac (GraphPad Software). Statistical differences were analyzed by parametric unpaired 2-tailed Student's $t$ test or 1-way ANOVA followed by Dunnett's test as indicated. $P$ values less than 0.05 were considered statistically significant. ${ }^{*} P<0.05$, ${ }^{* *} P<0.01, * * * P<0.001$.

\section{Author contributions}

AK designed the research studies, conducted experiments, acquired and analyzed data, and wrote the manuscript. SPC, FA, VM, GA, CS, and JCB conducted experiments, acquired and analyzed data. CC, VP, AP, EB, JPR, and ODC analyzed data. MB and CR provided patient blood samples. MB and PJL analyzed data and wrote the manuscript. CVD and DB were responsible for conception and supervision of the study and wrote the manuscript. All authors corrected draft versions and approved the final version of the manuscript. 


\section{Acknowledgments}

The authors wish to thank the patient who participated in this study and the Animal Platform, CRP2 UMS 3612 CNRS - US25 Inserm-IRD - Faculté des Sciences Pharmaceutiques et Biologiques, Université Paris Descartes, and Muriel Andrieu and Karine Labroquère of the Cochin Cytometry and Immunobiology Facility for using the BD LSR II cytometer and BD Canto II. This study was financially supported by grants from INSERM and Agence Nationale de la Recherche (ANR 11 BSV1-010-01 to C.V. Denis).

Address correspondence to: Alexandre Kauskot, INSERM U1176, Hémostase-Inflammation-Thrombose, Hôpital Kremlin Bicêtre, 80 rue du Général Leclerc, 94276 Le Kremlin-Bicêtre Cedex, Paris, France. Phone: 33.0.1.49.59.56.16; E-mail: alexandre.kauskot@inserm.fr.

1. Yang N, et al. Cofilin phosphorylation by LIM-kinase 1 and its role in Rac-mediated actin reorganization. Nature. 1998;393(6687):809-812.

2. Mizuno K. Signaling mechanisms and functional roles of cofilin phosphorylation and dephosphorylation. Cell Signal. 2013;25(2):457-469

3. Junt T, et al. Dynamic visualization of thrombopoiesis within bone marrow. Science. 2007;317(5845):1767-1770

4. Hartwig J, Italiano J. The birth of the platelet. J Thromb Haemost. 2003;1(7):1580-1586.

5. Italiano JE, Lecine P, Shivdasani RA, Hartwig JH. Blood platelets are assembled principally at the ends of proplatelet processes produced by differentiated megakaryocytes. J Cell Biol. 1999;147(6):1299-1312.

6. Hartwig JH, Italiano JE. Cytoskeletal mechanisms for platelet production. Blood Cells Mol Dis. 2006;36(2):99-103.

7. Thon JN, et al. Cytoskeletal mechanics of proplatelet maturation and platelet release. J Cell Biol. 2010;191(4):861-874

8. Bender M, et al. ADF/n-cofilin-dependent actin turnover determines platelet formation and sizing. Blood. 2010;116(10):1767-1775.

9. Zimmerman TS, Dent JA, Ruggeri ZM, Nannini LH. Subunit composition of plasma von Willebrand factor. Cleavage is present in normal individuals, increased in IIA and IIB von Willebrand disease, but minimal in variants with aberrant structure of individual oligomers (types IIC, IID, and IIE). J Clin Invest. 1986;77(3):947-951.

10. Casari C, et al. Accelerated uptake of VWF/platelet complexes in macrophages contributes to VWD type 2B-associated thrombocytopenia. Blood. 2013;122(16):2893-2902.

11. Saba HI, Saba SR, Dent J, Ruggeri ZM, Zimmerman TS. Type IIB Tampa: a variant of von Willebrand disease with chronic thrombocytopenia, circulating platelet aggregates, and spontaneous platelet aggregation. Blood. 1985;66(2):282-286.

12. Berrou E, et al. Apoptotic platelet events are not observed in severe von Willebrand disease-type 2B mutation p.V1316M. PLoS One. 2015;10(12): $\mathrm{e} 0143896$.

13. Federici $\mathrm{AB}$, et al. Clinical and molecular predictors of thrombocytopenia and risk of bleeding in patients with von Willebrand disease type 2B: a cohort study of 67 patients. Blood. 2009;113(3):526-534.

14. Nurden $\mathrm{P}$, et al. Impaired megakaryocytopoiesis in type $2 \mathrm{~B}$ von Willebrand disease with severe thrombocytopenia. Blood. 2006;108(8):2587-2595.

15. Poon MC, Rand ML, Jackson SC. 2B or not to be--the 45-year saga of the Montreal Platelet Syndrome. Thromb Haemost. 2010;104(5):903-910.

16. Dunois-Lardé C, Capron C, Fichelson S, Bauer T, Cramer-Bordé E, Baruch D. Exposure of human megakaryocytes to high shear rates accelerates platelet production. Blood. 2009;114(9):1875-1883.

17. Adam F, et al. A genetically-engineered von Willebrand disease type 2B mouse model displays defects in hemostasis and inflammation. Sci Rep. 2016;6:26306.

18. Chandraratne S, et al. Critical role of platelet glycoprotein iba in arterial remodeling. Arterioscler Thromb Vasc Biol. 2015;35(3):589-597.

19. Strassel C, et al. Intrinsic impaired proplatelet formation and microtubule coil assembly of megakaryocytes in a mouse model of Bernard-Soulier syndrome. Haematologica. 2009;94(6):800-810.

20. Larson MK, Watson SP. Regulation of proplatelet formation and platelet release by integrin alpha IIb beta3. Blood. 2006;108(5):1509-1514.

21. Pietrzyk-Nivau A, et al. Three-dimensional environment sustains hematopoietic stem cell differentiation into platelet-producing megakaryocytes. PLoS One. 2015;10(8):e0136652

22. Schulze H, et al. Characterization of the megakaryocyte demarcation membrane system and its role in thrombopoiesis. Blood. 2006;107(10):3868-3875.

23. Sui Z, Nowak RB, Sanada C, Halene S, Krause DS, Fowler VM. Regulation of actin polymerization by tropomodulin-3 controls megakaryocyte actin organization and platelet biogenesis. Blood. 2015;126(4):520-530.

24. Antkowiak A, et al. Cdc42-dependent F-actin dynamics drive structuration of the demarcation membrane system in megakaryocytes. J Thromb Haemost. 2016;14(6):1268-1284.

25. Malarkannan S, et al. IQGAP1: a regulator of intracellular spacetime relativity. J Immunol. 2012;188(5):2057-2063.

26. Estevez B, et al. LIM kinase-1 selectively promotes glycoprotein Ib-IX-mediated TXA2 synthesis, platelet activation, and thrombosis. Blood. 2013;121(22):4586-4594.

27. Bernard O. Lim kinases, regulators of actin dynamics. Int J Biochem Cell Biol. 2007;39(6):1071-1076.

28. Chang Y, et al. Proplatelet formation is regulated by the Rho/ROCK pathway. Blood. 2007;109(10):4229-4236.

29. Casari C, et al. von Willebrand factor mutation promotes thrombocytopathy by inhibiting integrin $\alpha \operatorname{IIb} \beta 3$. J Clin Invest. 2013;123(12):5071-5081.

30. Nurden P, et al. Abnormal VWF modifies megakaryocytopoiesis: studies of platelets and megakaryocyte cultures from patients 
with von Willebrand disease type 2B. Blood. 2010;115(13):2649-2656.

31. Cramer EM, et al. Uncoordinated expression of fibrinogen compared with thrombospondin and von Willebrand factor in maturing human megakaryocytes. Blood. 1989;73(5):1123-1129.

32. Pronk CJ, et al. Elucidation of the phenotypic, functional, and molecular topography of a myeloerythroid progenitor cell hierarchy. Cell Stem Cell. 2007;1(4):428-442.

33. Eckly A, et al. Biogenesis of the demarcation membrane system (DMS) in megakaryocytes. Blood. 2014;123(6):921-930.

34. Avanzi MP, Goldberg F, Davila J, Langhi D, Chiattone C, Mitchell WB. Rho kinase inhibition drives megakaryocyte polyploidization and proplatelet formation through MYC and NFE2 downregulation. Br J Haematol. 2014;164(6):867-876.

35. Pleines I, et al. Defective tubulin organization and proplatelet formation in murine megakaryocytes lacking Rac1 and Cdc42. Blood. 2013;122(18):3178-3187.

36. Bury L, Falcinelli E, Chiasserini D, Springer TA, Italiano JE, Gresele P. Cytoskeletal perturbation leads to platelet dysfunction and thrombocytopenia in variant forms of Glanzmann thrombasthenia. Haematologica. 2016;101(1):46-56.

37. Kile BT, et al. Mutations in the cofilin partner Aip1/Wdr1 cause autoinflammatory disease and macrothrombocytopenia. Blood 2007;110(7):2371-2380

38. Balduini A, et al. Adhesive receptors, extracellular proteins and myosin IIA orchestrate proplatelet formation by human megakaryocytes. J Thromb Haemost. 2008;6(11):1900-1907.

39. Suzuki A, et al. RhoA is essential for maintaining normal megakaryocyte ploidy and platelet generation. PLoS One. 2013;8(7):e69315.

40. Post A, Pannekoek WJ, Ponsioen B, Vliem MJ, Bos JL. Rap1 Spatially controls ArhGAP29 to inhibit Rho signaling during endothelial barrier regulation. Mol Cell Biol. 2015;35(14):2495-2502.

41. Mariana G, et al. Evaluation of the effectiveness of DDAVP in surgery and in bleeding episodes in haemophilia and von Willebrand's disease. A study on 43 patients. Clin Lab Haematol. 1984;6(3):229-238.

42. Federici AB, Castaman G, Mannucci PM, Italian Association of Hemophilia Centers (AICE). Guidelines for the diagnosis and management of von Willebrand disease in Italy. Haemophilia. 2002;8(5):607-621.

43. Boland S, et al. Design, synthesis and biological characterization of selective LIMK inhibitors. Bioorg Med Chem Lett. 2015;25(18):4005-4010.

44. Prudent R, et al. Pharmacological inhibition of LIM kinase stabilizes microtubules and inhibits neoplastic growth. Cancer Res. 2012;72(17):4429-4439.

45. Harrison BA, et al. Novel class of LIM-kinase 2 inhibitors for the treatment of ocular hypertension and associated glaucoma. JMed Chem. 2009;52(21):6515-6518.

46. Dai YP, Bongalon S, Tian H, Parks SD, Mutafova-Yambolieva VN, Yamboliev IA. Upregulation of profilin, cofilin-2 and LIMK2 in cultured pulmonary artery smooth muscle cells and in pulmonary arteries of monocrotaline-treated rats. Vascul Pharmacol. 2006;44(5):275-282.

47. Vorster PJ, et al. LIM kinase 1 modulates cortical actin and CXCR4 cycling and is activated by HIV-1 to initiate viral infection. J Biol Chem. 2011;286(14):12554-12564.

48. Poirault-Chassac S, et al. Notch/Delta4 signaling inhibits human megakaryocytic terminal differentiation. Blood. 2010;116(25):5670-5678.

49. Schulze H. Culture of murine megakaryocytes and platelets from fetal liver and bone marrow. Methods Mol Biol. 2012;788:193-203.

50. Schindelin J, et al. Fiji: an open-source platform for biological-image analysis. Nat Methods. 2012;9(7):676-682. 